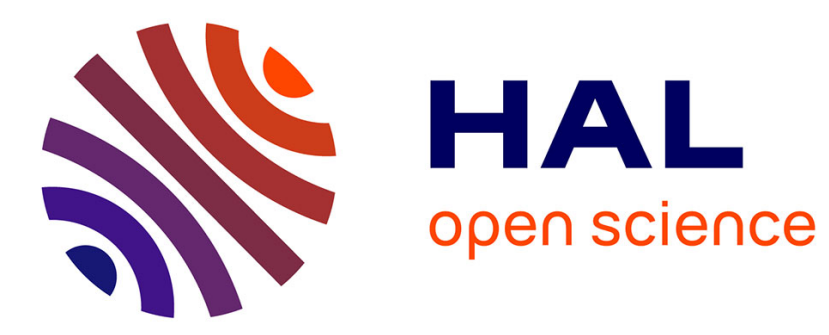

\title{
Ultrafast radial modulation imaging
}

Pauline Muleki Seya, Kailiang Xu, Tanter Mickael, Olivier Couture

\section{To cite this version:}

Pauline Muleki Seya, Kailiang Xu, Tanter Mickael, Olivier Couture. Ultrafast radial modulation imaging. IEEE Transactions on Ultrasonics, Ferroelectrics and Frequency Control, 2019, 10.1109/TUFFC.2019.2949046 . hal-02345642

\section{HAL Id: hal-02345642 \\ https://hal.science/hal-02345642}

Submitted on 4 Nov 2019

HAL is a multi-disciplinary open access archive for the deposit and dissemination of scientific research documents, whether they are published or not. The documents may come from teaching and research institutions in France or abroad, or from public or private research centers.
L'archive ouverte pluridisciplinaire HAL, est destinée au dépôt et à la diffusion de documents scientifiques de niveau recherche, publiés ou non, émanant des établissements d'enseignement et de recherche français ou étrangers, des laboratoires publics ou privés. 


\title{
Ultrafast radial modulation imaging
}

\author{
P. Muleki-Seya, K. Xu, M. Tanter Member, IEEE, and O. Couture, Member, IEEE
}

\begin{abstract}
Radial modulation imaging improves the detection of microbubbles at high frequency by using a dual ultrasonic excitation. However, the synchronization between the imaging pulses is non-trivial because microbubbles need to be interrogated in the compression and the rarefaction phase and the time-delay difference from dispersion has to be corrected. To address these issues, we propose the use of ultrafast radial modulation imaging (uRMI). In this technique, a beat frequency between the modulation pulse (around $1 \mathrm{MHz}$ ) and the ultrafast pulse-repetition frequency was exploited to separate microbubbles from tissue phantom in-vitro. This led to a modulated images set in the spectral domain of the slow-time that may then be demodulated through a digital lock-in amplifier to retrieve the contrast image. Ultrafast RMI, applied on a flow phantom with microbubbles, provided a contrast-to-tissue ratio from 7.2 to $14.8 \mathrm{~dB}$ at $15 \mathrm{MHz}$. For flow speed lower than $0.05 \mathrm{~mL} / \mathrm{min}$, uRMI $(16 \mathrm{~dB})$ provided a better contrast-to-tissue ratio than other techniques: SVD spatiotemporal filter $(11 \mathrm{~dB})$, amplitude modulation $(9 \mathrm{~dB})$ or microbubbles disruption $(6 \mathrm{~dB})$ or. This technique may then be suitable to improve the detection of targeted microbubbles, in ultrasound molecular imaging applications, and the detection of extremely slow microbubbles moving in the finest vessels in ultrasound localization microscopy.
\end{abstract}

Index Terms - Contrast Imaging, Microbubbles, Radial modulation, Ultrafast imaging.

\section{INTRODUCTION}

OST major causes of death are linked to diseases that affect, in some way, the microvasculature. Cancer grows and spreads with the help of angiogenesis, arteriosclerosis often involves the progression of the vaso vasorum and ischemic stroke implies the blockage of a significant number of cerebral vessels of all sizes. In these areas, ultrasound imaging is a key diagnostic tool, mainly because Doppler is sensitive to blood flow [1].

Recent advances in ultrafast imaging, based on plane-wave emission and parallel beamforming [2,3], have increased the sensitivity of Doppler by more than an order of magnitude $[4,5]$. For instance, it is now possible to assess the function of the brain

This work was supported principally by Agence Nationale de la Recherche, within the project ANR Tremplin. This project has received funding from the European Research Council under the European Union Horizon H2020 programme/ERC Consolidator grant agreement No 772786-ResolveStroke. 
with ultrasound through the neuro-vascular coupling $[6,7]$. However, the finest vessels, smaller than the ultrasound wavelength, are still undetectable since red blood cells are poor scatterers that are moving insufficiently to create a detectable decorrelation.

To improve the sensitivity of ultrasound to the microvasculature, microbubbles were introduced as ultrasound contrast agents [8]. The current generation of agents is formed by bubbles of perfluorocarbon gases, a few microns in size, which remain in the vasculature after being intravenously injected [9]. Their detection is based not only on the impedance mismatch between gas and liquid but also due to their resonance in the $\mathrm{MHz}$ range. Moreover, their oscillation becomes highly nonlinear in the diagnostic range of ultrasonic pressures and they can even be disrupted with sufficiently high-pressure pulses [10, 11].

Several detection techniques have been designed to separate microbubbles from the tissue. Microbubbles can emit harmonic components, as well as subharmonics and ultraharmonics, which falls at fraction multiple of the fundamental frequency. Harmonic imaging modulation (denoted amplitude modulation AM) methods, for which echoes from pulses of different amplitudes are combined, are also in use [12,13]. They can also be combined with pulse inversion (PI) methods [14]. Both these techniques were already implemented with ultrafast scanner [15-17]. To minimize the signal from tissue, another possibility is subharmonic imaging, as the nonlinear signal generated by tissue contains only harmonics components $[18,19]$. The generation of very high ultraharmonics can also be implemented to separate microbubbles from tissue, allowing the reception of very high frequencies, resulting in excellent resolution as in ultrasound angiography [20]. These techniques have inherent limitations associated with the fact that they rely on the strong nonlinear oscillations of microbubbles, putting microbubbles at risk of disruption. Moreover, they often lock the emission pulse frequency to the bubble resonance frequency (below $10 \mathrm{MHz}$ ) and they consequently limit image resolution.

Other microbubbles detection approaches have been used. The oldest [8] is the detection of the microbubble decorrelation through their motion. It was recently exploited for ultrasound localization microscopy [21-23] to detect microbubbles from the tissue before the localization step which improved the resolution by an order of magnitude as compared to the diffraction limit. For this, spatiotemporal filters were implemented on stacks of ultrafast imaging. Singular-value decomposition (SVD) of large ensembles of ultrafast images was proved to be superior to nonlinear techniques for flow within the $\mathrm{mm} / \mathrm{s}$ range, both in-vitro and the clinic [24]. However, we have yet to detect microbubbles moving well below $1 \mathrm{~mm} / \mathrm{s}$ even at higher imaging frequencies. Microbubbles moving at these flow speed in the finest vessels may not be detected by spatiotemporal filtering. Spatiotemporal filtering is also poorly suited for ultrasound molecular imaging, where microbubbles are attached to targets through ligands and, consequently, remain still [25-27]. For these two applications, there is a need for an imaging technique allowing the detection of fixed or slowly moving microbubbles at high frequency.

An interesting approach to detect microbubbles is to exploit, not their harmonic nonlinearities or their motion, but their high compressibility and eventually their nonlinear elasticity [28]. Radial modulation imaging (RMI), a particular application of a dual 
frequency band technique is based on the observations of an increase of the microbubbles' amplitude in radio-frequency data in presence of superimposed ultrasound excitations, say $10 \mathrm{MHz}$ and $0.5 \mathrm{MHz}$ [29]. In RMI, a low-frequency pulse (the modulation pulse) induces oscillation of microbubbles and modify its size. The microbubbles are simultaneously interrogated using a high-frequency pulse (the imaging pulse). One implementation of RMI consists of synchronizing two successive short imaging pulses such that they reach the microbubble when it is in a compressed and an expanded state, induced by the modulation pressure wave. By subtracting successive imaging lines, this approach results in an image of microbubbles, in which tissue scattering is suppressed because it is minimally affected by the modulation pulse. Radial modulation on a single microbubble affects microbubble's radius and echoes' amplitude [30]. The predominant effect in RMI was found to be microbubble size and not microbubble resonance or compression-only effect [31]. Simulations suggested that a $10 \%$ variation of bubble diameter induced by the modulation excitation is sufficient for radial modulation imaging [32].

In 2006, on a flow phantom with Definity contrast agent circulating, Shariff and al. [33], showed a 35 dB microbubbles signal enhancement using RMI. Angelsen et al. $[34,35]$ demonstrated numerically that SURF imaging (RMI with a frequency separation between the modulation and the imaging pulses of a factor of 10) with correction of the dispersion of the imaging pulse by the modulation pulse provided higher contrast than PI. This team also pointed out that in the presence of the manipulation pulse, the imaging pulse presented a distortion which was different than for conventional harmonic distortion [36]. This technique showed promising results in vitro with a $20 \mathrm{MHz}$ single-element intravascular ultrasound catheter [37] and in vivo for pig kidney with a bolus injection of Sonovue [38]. Nevertheless, several issues remain to be solved with RMI. For instance, the synchronization between the imaging pulses is non-trivial for the classical RMI process because microbubbles need to be interrogated in the compression and the rarefaction phase and not between them (ie, in nodes of the modulation pressure). Moreover, the time-delay difference corresponding to the dispersion of the imaging pulse by the modulation pulse must be corrected [34, 35].

In this study, we propose to simplify RMI and solve the synchronization issue by sampling the oscillations of microbubbles induced by the modulation pulse with ultrafast imaging. The use of ultrafast imaging will allow in one hand to increase the number of microbubble modulation-states and in another hand to improve the efficiency of filtering in the slow time by increasing the number of images. We hypothesize that the use of ultrafast imaging to sample the oscillations of the microbubbles in RMI could improve its sensitivity and simplify the process. First, by easily allowing the sampling of several modulation states $(3,4,5$ or 10$)$, it would overcome the synchronization issue. Indeed, there is no risk for an acquisition to be performed at the nodes of the modulation oscillation when a 3 modulation-states sequence is performed. Second, comparable to ultrasensitive Doppler [39], we suggest that the modulation frequency can be filtered more efficiently on long ensembles of images, opening the possibilities for highly-specific filtering methods, such as a lock-in amplifier. Moreover, with this new process, there is no necessity to correct for the dispersion between the high-frequency pulse and the modulation pulse. We assume that RMI combined with ultrafast 
acquisitions will be more appropriate to detect fixed and extremely slowly moving microbubbles than other techniques to detect microbubble at high frequency.

We describe an implementation of ultrafast RMI (uRMI) where a beat frequency between the modulation pulse and the ultrafast pulse-repetition frequency is exploited to separate microbubbles from tissue phantom in-vitro. In this way, recording microbubbles in different states of the modulation period translates to a modulated images set in the spectral domain of the slow-time. The microbubble images may then be evaluated by demodulation of the images through a lock-in amplifier. We evaluate uRMI in-vitro at high imaging frequencies $(15 \mathrm{MHz})$ where nonlinear methods tend to be less efficient because microbubbles are off-resonance. Different parameters' effect is explored: the amplitude of the modulation pulse, the flow speed, the number of sampled modulation states and the number of angles of imaging plane waves acquisitions. Then, this method is compared with other microbubbles detection techniques, amplitude modulation, microbubble disruption, and SVD spatiotemporal filter.

\section{MATERIAL AND METHODS}

\section{A. Simulations}

The microbubbles backscatter signals in response to amplitude modulation (AM), bubbles disruption and classical RMI imaging were calculated using thin-shelled microbubble oscillation theory at $15 \mathrm{MHz}$. The simulation package Bubblesim [40] was used with the Rayleigh-Plesset equation with radiation damping. Shell viscosity was chosen as 0.5 Pa.s, shear modulus as $20 \mathrm{MPa}$, and shell thickness as $4 \mathrm{~nm}$, corresponding to thin-shelled lipid-encapsulated microbubbles [40]. BubbleSim assumes a viscoelastic shell. A more complete description of oscillating Sonovue microbubbles would have included the buckling effect modelled by Marmottant [28]

Pulses of 1 cycle with amplitude pressure from 200 to $800 \mathrm{kPa} 15 \mathrm{MHz}$ were simulated for a microbubble of $1.25 \mu \mathrm{m}$ radius (corresponding to the mean radius of microbubble present in Sonovue [41], $2.5 \mu \mathrm{m}$ radius and $3.7 \mu \mathrm{m}$ radius (corresponding to the resonant radius at $1 \mathrm{MHz}$ from simulations). These radii were present in Sonovue as more than $90 \%$ of the bubbles are smaller than $4 \mu \mathrm{m}$ in radius [41]. The scattered pressure from a microbubble in AM mode, $P_{S_{A M}}$, was evaluated as:

$$
P_{S_{A M}}(a)=\max \left(P_{S}(t, a)-2 P_{S}\left(t, \frac{a}{2}\right)\right)
$$

where $P_{S}$ denoted the microbubble scattered pressure, and $a$ is the pressure amplitude of the 15-MHz pulse.

The scattered pressure from the microbubble disruption, $P_{S_{D}}$, represented by the complete disappearance of the microbubble echo between two images was evaluated as: 


$$
P_{S_{D}}(a)=\max \left(P_{S}(t, a)\right)
$$

To evaluate the scattered pressure from a microbubble in RMI, $P_{S_{R M I}}$, the microbubble radius in rarefaction and compression state at $1 \mathrm{MHz}$ for pressure amplitude of 10,30, 50 and $100 \mathrm{kPa}$ were estimated first for a 25-cycles excitation. Then, the scattered pressure at $15 \mathrm{MHz}$ for the different pressure amplitudes (between 200 and $800 \mathrm{kPa}$ ) for this minimum and maximum radius were evaluated for a 1-cycle excitation. The scattered pressure for RMI is evaluated as:

$$
P_{S_{R M I}}(a)=\max \left(P_{s}\left(t, a, R_{\max }\right)-P_{s}\left(t, a, R_{\min }\right)\right)
$$

where $R_{\text {max }}$ and $R_{\text {min }}$ denoted the maximum and minimum radius estimated at compression and rarefaction state, respectively.

\section{B. Ultrafast radial modulation imaging}

Sonovue microbubbles (Bracco), diluted at 1/3000 (with an approximate concentration of 30000 to $16 \times 10^{4} \mathrm{microbubbles} / \mathrm{mL}$ ) circulated at a flow rate between 0 and $20 \mathrm{~mL} / \mathrm{min}$ in a $2 \mathrm{~mm}$ diameter vessel phantom by syringe pump or by gravity. The flow rate was determined by measuring the volume of outflow over 1 minute of flow. To guarantee the absence of flow when required, the flow was stopped 30 seconds before the acquisition. This microbubble wall-less vessel phantom was included in a $4 \mathrm{~cm}$ thick $5 \%$ (w/w) agar phantom comprising 1\% (w/w) sigma-cell (20 $\mu \mathrm{m}$ particles, Sigma-Aldrich, Saint Louis, MO) to mimic tissue scattering (Fig. 1). A 15-MHz linear transducer array (Vermon, France, 128 elements, pitch: 0.11 mm and bandwidth: 9-24 MHz at $-10 \mathrm{~dB}$ ) was placed above the agar phantom and a 1-MHz modulation transducer was positioned next to it, in a way that their pressure fields overlapped at the vessel phantom location. The 1-MHz modulation transducer was conceived in the laboratory with a piezocomposite material of $23 \mathrm{~mm}$ by $10 \mathrm{~mm}$ in size (Smart Materials, Dresden, Germany), air-backed, inserted in a 3-D printed plastic structure and soldered to a coaxial cable. It was sealed with hot-melt adhesive. The 1-MHz modulation transducer and the 15 $\mathrm{MHz}$ probe were inserted in a 3-D printed plastic holder in order to keep a $45^{\circ}$ angle between them.

High-frequency imaging was performed with the 15-MHz transducer driven by a 256 Vantage system (Verasonics, Kirkland, WA). Plane waves with 1 cycle pulse at $15 \mathrm{MHz}$ with 1 to 7 angles (between $-10^{\circ}$ and $10{ }^{\circ}$ ) were generated. A pulse-repetition-frequency $(\mathrm{PRF})$ of $1 / 60 \mu$ s was chosen. The imaging peak-negative pressure was $300 \mathrm{kPa}(\mathrm{MI}=0.08)$. Ultrafast radial-modulation imaging consisted of addressing the microbubbles at different stages of their oscillations. Several numbers of microbubble modulation-states were tested, each fitted within the same imaging angle. For example, the sequence for a 4 modulation-states and 3 angles excitation was [state 1 steering 1 , state 2 steering 1 , state 3 steering 1 , state 4 steering 1 , state 1 
steering 2 , etc], adding to a 12 pulses acquisition, with a frame rate of $1 /(12 * 60) \mu$ s. The more modulation-states or the more angles used, the lower was the frame rate. The low-frequency was generated, via the 1-MHz transducer, by a function generator (DG1022A, RIGOL, Beaverton, OR, USA). The low-frequency was chosen in a way that high-frequency pulses every $60 \mu \mathrm{s}$ reached microbubble in 3, 4, 5 or 10 modulation-states (see Fig. 2) and the corresponded frequencies were $0.9889,1.0042,1.0033$ and $1.0017 \mathrm{MHz}$, respectively. The amplitude pressures of the low-frequency were between 10 and $35 \mathrm{kPa}$ as measured by both a calibrated hydrophone (HNC-0400, Onda, Sunnyvale, CA, USA) and an optical interferometer (homemade from [42]).

For each measurement, 1000 images were acquired with the imaging transducer at a sampling frequency of $31.25 \mathrm{MHz}$. The radio-frequency data for each angle were separated and beamformed.

To increase the sensitivity of the system to the narrowband radial modulation, a lock-in amplifier was implemented on the channel data in the slow time. It corresponds to a digital amplifier implemented in image processing using Matlab. This demodulation process consisted of filtering each channel data around the modulation frequency using a bandpass filter (Butterworth filter with cut-off frequencies of 0.9 and 1.1 times the modulation frequency with an order of 48 ). The modulation frequency is the sampling frequency in the slow time divided by the number of microbubble modulation-states. Then, the filtered signals were multiplied with a sinusoidal signal at the modulation frequency before to be filtered by a lowpass filter in the slow time (Butterworth filter with a cut-off frequency of 0.1 times the sampling frequency with an order of 48 ).

Reconstruction of images was performed by combining coherently the summing angles. In the case of 5 and 10 microbubble modulation-states, the beamformed images were also filtered at the modulation frequency multiplied by 2 (for 5 and 10 microbubble modulation-states), 3 (for 10 microbubble modulation-states) and 4 (for 10 microbubble modulation-states). For these two microbubble modulation-states numbers, the reconstructed set of images corresponded to the product of the filtered images for the modulation frequency and its multiples. These uRMI images filtered at several frequencies were named multi-band uRMI.

The amplitude of the signal in the agar and the vessel phantom was estimated for the sum in intensity of these 1000 processed images. Acquisitions were repeated 3 times.

\section{Other techniques}

The uRMI results were compared with other techniques to detect microbubbles: amplitude modulation, microbubbles disruption, and SVD spatiotemporal filter.

\section{1) Amplitude modulation}

A Verasonic plane-waves amplitude modulation sequence with the $15-\mathrm{MHz}$ transducer was implemented. In this sequence for each angle of each image with an amplitude of $300 \mathrm{kPa}, 3$ pulses were defined: a first 1 cycle $15 \mathrm{MHz}$ pulses with all elements, a 
second with only odd elements and a third with only even elements. The amplitude modulation image was estimated by subtracting the RF data from odd and even elements to the image with all elements before beamforming these different images. The PRF was $1 /(3 * 60) \mu \mathrm{s}$, the same as PRF from uRMI imaging for 3 angles. One thousand images were acquired and the amplitude of the signal in the agar and the vessel phantom was estimated for the sum in intensity of these 1000 images. Acquisitions were repeated 3 times.

\section{2) Microbubble disruption}

At best, disruption imaging should provide the same contrast as the difference in signal between a microbubble solution and water. To evaluate the amplitude of the signal due to microbubbles, 1000 images were acquired before and after microbubbles injection in the vessel phantom. The amplitude of the signal in the agar and the vessel phantom was estimated on the sum in intensity of the images before microbubbles injection and on the sum in intensity of images after microbubble injection. The microbubble contrast is the difference between the intensity in the vessel when it is filled with microbubbles and when it is filled with water. The ROI used were two times smaller than for amplitude modulation, uRMI or SVD filtering to limit the presence of artifacts in the vessel ROI. In comparison, the other techniques naturally limit the amplitude of the phantom.

\section{3) SVD filter}

The same sequence as the ultrafast RMI sequence without the $1 \mathrm{MHz}$ modulation pulse was used. After the image acquisition, these images were beamformed and filtered with an SVD filter to remove eigenvalues corresponding to tissue and noise (eigenvalues between 3/1000 and 120/1000 were kept). The amplitude of the signal in the agar and the vessel phantom was estimated for the sum in intensity of these 1000 images. Acquisitions were repeated 3 times.

\section{RESULTS}

\section{A. Simulations}

In this section, the simulated scattered pressure from a microbubble from RMI, AM and microbubble disruption is evaluated to determine which technique provides the higher scattered pressure as a function of amplitude pressure and for different microbubble radius.

The simulation results are presented in Fig. 3. The scattered pressure for RMI varies with the microbubble-radius. This scattered pressure is higher for the $3.7 \mu \mathrm{m}$ microbubble radius, the resonant radius at $1 \mathrm{MHz}$, followed by the scattered pressure for the 2.5 $\mu \mathrm{m}$ microbubble radius. For the $1.25 \mu \mathrm{m}$ radius, the RMI, for the amplitude pressure of the $1 \mathrm{MHz}$-excitation of 10 and $30 \mathrm{kPa}$, present scattered pressure lower than AM; whereas the RMI, for amplitude pressure of the $1 \mathrm{MHz}$-excitation of 50 and $100 \mathrm{kPa}$, present scattered pressure higher than AM. The scattered pressure from RMI and amplitude modulation is much lower than from 
microbubbles disruption. For the $2.5 \mu \mathrm{m}$ radius, the scattered pressure for RMI is higher than AM imaging but lower than microbubbles disruption. For the $3.7 \mu \mathrm{m}$ radius, the scattered pressure for RMI, for amplitude pressure of the $1 \mathrm{MHz}$-excitation of 50 and $100 \mathrm{kPa}$, is even higher than the scattered pressure from microbubbles disruption.

\section{B. Ultrafast radial modulation imaging}

In this section, the effect of different parameters on the contrast-to-tissue ratio obtained with uRMI are evaluated to determine the limits of this technique.

After an uRMI acquisition, a set of 1000 ultrafast Bmode images were obtained. From these Bmode images, the vessel phantom and a phantom location were determined. The selected vessel and phantom region-of-interest had the same dimensions and the same depth. The temporal fast Fourier transform of these Bmode image sets, averaged at the vessel phantom location, was calculated. Examples of the frequency spectrum in slow time from these measured data (normalized by the sampling frequency of the acquisition) are presented in Fig. 4 for several microbubble modulation-states. For a 3 microbubble modulation-states, peaks are present in the spectrum due to the modulation at $\mathrm{f} / \mathrm{fs}=0.33$ and 0.66 . For a 4 microbubble modulation-states, peaks are present in the spectrum due to the modulation at $\mathrm{f} / \mathrm{fs}=0.25,0.5$ and 0.75 . For a 5 microbubble modulation-states, peaks are present in the spectrum due to the modulation at $\mathrm{f} / \mathrm{fs}=0.2,0.4,0.6$ and 0.8 . For a 10 microbubble modulation-states, peaks are present in the spectrum due to the modulation at $\mathrm{f} / \mathrm{fs}=0.1,0.2,0.3,0.4,0.5,0.6,0.7,0.8$ and 0.9 . Due to the number of microbubble modulation-states, a peak due to modulation was expected at $\mathrm{f} / \mathrm{fs}=0.33$ for 3 microbubble modulation-states, $\mathrm{f} / \mathrm{fs}=0.25$ for 4 microbubble modulation-states, $\mathrm{f} / \mathrm{fs}=0.2$ for 5 microbubble modulation-states and $\mathrm{f} / \mathrm{fs}=0.1$ for 10 microbubble modulation-states . The presence of other peaks may come from the interaction of a different configuration of microbubbles states (i.e for a 4 modulation-states, the peak at the normalized frequency 0.5 results from the interaction of state 1 and state 3 and/or state 2 and state 4 only).

The uRMI technique was applied in the presence and absence of microbubbles, with and without low-frequency excitation to evaluate their effect on the modulation and demodulation process. In absence of low-frequency excitation means that ultrafast imaging is the only ultrasonic excitation. Examples of microbubbles images acquired are presented in Fig. 5. In the presence of microbubbles and low-frequency excitation, microbubbles in the vessel phantom are observed in the image (Fig. 5(b)). When the sum of the 1000 images are used, the entire vessel phantom is well defined in the uRMI image (Fig. 5(c)). However, without microbubbles (Fig. 5(d) and (e)) or low-frequency excitation (Fig. 5(d) and (f)), microbubbles cannot be detected. The corresponding contrast-to-tissue ratios are listed in Table 1. In the presence of microbubbles, the contrast-to-tissue ratio in the Bmode image shows an increase of around $3 \mathrm{~dB}$. In the absence of low-frequency excitation, the contrast-to-tissue ratio for uRMI 
is close to $0 \mathrm{~dB}(0 \mathrm{~dB}$ is due to measurements hitting noise floor for both vessel and agar background). In the presence of low-frequency excitation, the contrast-to-tissue ratio for uRMI is $2.4 \mathrm{~dB}$ without microbubbles and $12.7 \mathrm{~dB}$ with microbubbles.

The effect of the low-frequency excitation pressure on the contrast-to-tissue ratio is presented in Fig. 6 for 4 microbubble modulation-states and 3 angles high-frequency excitation without flow and with a 1 and $3 \mathrm{~mL} / \mathrm{min}$ flow speed. The contrast-to-tissue ratio in the vessel phantom as compared to agar increases with low-frequency excitation pressure from 10 to 25 $\mathrm{kPa}$ and then is stable from 25 to $35 \mathrm{kPa}$ without flow. The same behavior was observed for 1 and $3 \mathrm{~mL} / \mathrm{min}$ flow speed (Fig. 6(a)). White artifacts (indicated by white arrows in Fig. 6(d-e)) corresponding to vertical lines mostly observed under the vessel) were observed only in uRMI images for a low-frequency pressure equal or superior at $25 \mathrm{kPa}$ (Fig. 6(b-e)). On the frequency spectrum at the vessel phantom location (Fig. $6(\mathrm{f}-\mathrm{g})$ ), the amplitude of the peak at the modulation frequency $(\mathrm{f} / \mathrm{fs}=0.25)$ increases with the amplitude of the $1 \mathrm{MHz}$ modulation. The main peak appears split for amplitude higher or equal to $25 \mathrm{kPa}$. For these flow speed, compounding angles' number and microbubble modulation-states' number, these results suggest that a pressure amplitude slightly lower than $25 \mathrm{kPa}$ should provide a good contrast-to-tissue ratio and prevent the presence of artifacts.

The effect of flow speed on the contrast-to-tissue ratio is presented in Figure 7 for 4 microbubbles modulation-states, a 3 angles high-frequency excitation and a $15 \mathrm{kPa}$ low-frequency excitation. The contrast-to-tissue ratio decreases slightly with the flow speed (Figure 7(a)). On the uRMI images, the signal in the center of the vessel phantom decreases when flow speed increases (Figure 7(b-e)) but there is always signal in the border of the vessel phantom for the different flow speed used. It may correspond to microbubbles in the boundary layer and at a lower speed than microbubbles in the center of the vessel phantom. When using an ROI at the center of the vessel phantom only (dotted ROI, 2 times smaller than the previous ROI), the contrast-to-tissue ratio is slightly higher for flow speed until $5 \mathrm{~mL} / \mathrm{min}$, and the decrease of contrast-to-tissue ratio with flow speed is more pronounced. The decrease of contrast-to-tissue ratio with flow speed is related to an evolution of the frequency spectrum at the vessel phantom location (Figure 7(f-g)): the amplitude of the peak at the modulation frequency ( $\mathrm{f} / \mathrm{fs}=0.25)$ decreases when flow speed increase and another peak with a higher frequency appears from $5 \mathrm{~mL} / \mathrm{min}$ with an amplitude and a center frequency increasing with the flow speed (from $\mathrm{f} / \mathrm{fs}=0.26$ at $5 \mathrm{~mL} / \mathrm{min}$ to $\mathrm{f} / \mathrm{fs}=0.32$ at $20 \mathrm{~mL} / \mathrm{min}$ ). For the experiment conditions, these compounding angles' number, microbubble modulation-states' number and amplitude of the low-frequency excitation, these results suggest that a flow speed equal or lower to $5 \mathrm{~mL} / \mathrm{min}$ should provide the best contrast-to-tissue ratios. Note that for a vessel in-plane, the flow speed threshold may be different due to higher Doppler shift.

The effect of the number of microbubble modulation-states on the microbubble contrast is presented in Fig. 8 for 3 angles high-frequency excitation and a $15 \mathrm{kPa}$ low-frequency excitation without flow and with a $3 \mathrm{~mL} / \mathrm{min}$ flow speed. Contrast-to-tissue ratios are similar with and without flow rate (Fig. 8(a)): the contrast-to-tissue ratio increases slightly with the number of microbubble modulation-states (from $10 \mathrm{~dB}$ to $12 \mathrm{~dB}$ for 3 and 10 microbubble modulation-states, respectively) and the use of the 
multiple of the modulation frequency allow to increase this contrast up to $17 \mathrm{~dB}$ for 5 microbubble modulation-states and up to 24 $\mathrm{dB}$ for 10 microbubble modulation-states. This increase of contrast-to-tissue ratio is observed on the uRMI and multi-band uRMI images (Fig. 8(b-e)). For these flow speed, compounding angles' number and amplitude of the low-frequency excitation, these results suggest that using a higher number of microbubble modulation-states and especially with multi-band uRMI should provide the best contrast-to-tissue ratios.

The effect of the number of angles in high-frequency acquisition on the contrast-to-tissue ratio is presented in Fig. 9 for 4 microbubble modulation-states and a $15 \mathrm{kPa}$ low-frequency excitation without flow and with a $3 \mathrm{~mL} / \mathrm{min}$ flow speed. Without flow, there was no effect of the number of angles in high-frequency acquisition but for a 1 or $3 \mathrm{~mL} / \mathrm{min}$ flow speed, the contrast-to-tissue ratio decreases with the number of angles (Fig. 9(a)). Individually the contrast of microbubbles in the vessel phantom and agar increases with the number of angles (Fig. 10(b)) without flow. This increase of agar amplitude is observed on the uRMI images (Fig. 9(b-e)). We wished to determine the reason behind the lack of increase of microbubbles contrast with the number of angles. Our two hypotheses were (1) an issue with angles compounding due to floating microbubbles and (2) an effect of RMI filtering. In this way, acquisitions with and without low-frequency excitation were recorded. The contrast-to-tissue ratio from these acquisitions was evaluated by subtracting the median image of each image set over time (Fig. 10(a)). In these conditions, there was an increase of contrast-to-tissue ratio with the number of angles (Fig. 9(a)) with and without low-frequency excitation. Examples of frequency spectrums at the vessel phantom location are presented in Fig. 9(f-g). In these spectrums, the amplitude of the peak at the modulation frequency $(\mathrm{f} / \mathrm{fs}=0.25)$ increases with the number of angles and another peak with a higher frequency appears until 3 angles with an amplitude and a center frequency increasing with the number of angles (from $\mathrm{f} / \mathrm{fs}=0.26$ for 3 angles to $\mathrm{f} / \mathrm{fs}=0.275$ for 7 angles). For these flow speed, microbubble modulation-states' number and amplitude of the low-frequency excitation, these results suggest that there is no need for using a high number of compounding angles to obtain a good contrast-to-tissue ratio.

\section{Comparison with other techniques}

In this section, the contrast-to-tissue ratio with uRMI and other techniques to detect microbubbles is evaluated without flow until moderate flows to determine if uRMI is more appropriate to detect fixed or very slowly moving microbubbles.

The contrast-to-tissue ratio was estimated with $\mathrm{AM}$ at $15 \mathrm{MHz}$, microbubble disruption and SVD spatiotemporal filtering and compared to the contrast-to-tissue ratio obtained with URMI for 4 modulation-states, 3 angles high-frequency excitation and a 23 $\mathrm{kPa}$ low-frequency excitation. The results are presented in Fig. 11 as well as examples of images obtained with these techniques. Contrast-to-tissue ratios obtained with uRMI for flow speed from 0 to $5 \mathrm{~mL} / \mathrm{min}$ were stable between 14 and $16 \mathrm{~dB}$. Contrast-to-tissue ratios obtained with SVD filter increases with the flow speed from around $11 \mathrm{~dB}$ (lower than the ratios obtained 
with uRMI until $0.05 \mathrm{~mL} / \mathrm{min}$ ) to $21 \mathrm{~dB}$ (higher than the ratios obtained with uRMI up to $0.6 \mathrm{~mL} / \mathrm{min}$ ). Contrast-to-tissue ratios obtained with amplitude modulation was between 8 and $10 \mathrm{~dB}$ and it was close to $6 \mathrm{~dB}$ for microbubbles disruption.

\section{DISCUSSION}

The objective of this study was first to describe the implementation of radial modulation with ultrafast imaging to simplify the RMI process and increase its sensitivity, and second to demonstrate that uRMI improves the detection of fixed or extremely slowly moving microbubbles at high-frequencies $(15 \mathrm{MHz})$. The acquisitions were performed in a vessel phantom where the pressure field of an ultrafast pulsed high-frequency excitation and a continuous low-frequency excitation overlapped. The images sets obtained were modulated in the slow time and the microbubbles images were extracted by demodulation with a lock-in amplifier. The number of microbubble modulation-states may be chosen by changing slightly the low-frequency continuous excitation frequency around $1 \mathrm{MHz}$. This way, the microbubble signal was observed as a beat frequency between the modulation frequency and the pulse-repetition-frequency.

In our experimental conditions, a contrast-to-tissue ratio from 7.2 to $14.8 \mathrm{~dB}$ was obtained with uRMI at $15 \mathrm{MHz}$. The contrast-to-tissue ratio increases with the amplitude of excitation of the low frequency and with the number of microbubbles modulation-states. It was stable with the number of high-frequency plane wave compounding angles and decreases with the flow speed. According to the results, there were two trade-offs to deal with to optimize the contrast-to-tissue ratio obtained with uRMI.

The first tradeoff was related to the amplitude of excitation of the low frequency. The contrast-to-tissue ratio increased with the pressure amplitude until a threshold around $(25 \mathrm{kPa})$ but at higher pressure amplitudes, artifacts appeared in uRMI images. These artifacts (Fig. 6(d-e)) corresponding to vertical lines were mostly observed under the vessel and may be the consequence of the modulation of the ultrasound signal after going through the vessel and so, to the coupling of the low and high ultrasound excitations. The presence of these artifacts was accompanied by a split of the peak at the modulation frequency on the frequency spectrum in the slow time (Fig. $6(\mathrm{~g})$ ), meaning that the pressure amplitude may be optimized by the apparition of this split. The presence of artifacts was also highlight by Shariff et al. [33], which associated them to a consequence of a coupling between the modulation and imaging frequencies possibly resulting from disruption of the bubbles, and by Cherin et al. [32], which associate them to the generation of harmonics by bubbles strongly resonating at the modulation frequency.

The second trade-off was related to the microbubbles decorrelation, an inherent limitation of RMI techniques, which led to a decrease in the contrast-to-tissue ratio. This microbubble decorrelation may be a consequence of a too high flow speed or a too low frame rate due to a large number of imaging compounding angles or microbubble modulation-states. 
In presence of flow speed higher than $5 \mathrm{~mL} / \mathrm{min}$, the contrast-to-tissue ratio tended to decrease (Fig. $7(\mathrm{a})$ ), which was characterized by the presence of another broader peak at a higher frequency than the modulation frequency on the frequency spectrum (Fig. 7(g)). This other broader peak may correspond to a pulsed-Doppler shift induced by the flow. This shift of energy away from the main band due to the decorrelation of microbubbles, and eventually to their displacement, led to a reduction of the contrast-to-tissue ratio.

When the number of microbubbles modulation-states increased, the contrast-to-tissue ratio increased slightly (Figure 8(a)). It seemed that using more microbubbles modulation-states provided more information about the microbubbles. However, using more microbubbles modulation-states led also to a decrease in the frame rate. This was seen on the slow-time spectra where the Doppler-shifted modulation band became more prominent with higher flows (Figure 8(f)). However, for a higher flow speed, one may expect that using more microbubbles modulation-states, and so a lower frame rate, should lead to a decrease in the contrast-to-tissue ratio.

Without flow, the number of angles did not affect the contrast-to-tissue ratio (Fig. 9(a)). Classically, the contrast of an image should increase with the number of angles [3]. Individually, the amplitude in the phantom and the vessel increased with the number of angles (Fig. 10(b)). This parallel increase implies that little gain is observed in the contrast-to-tissue ratio with more compounding angles. Conversely, in the presence of flow, the contrast-to-tissue ratio decreased with the number of angles (Figure 9(a)). This decrease was related to the increase of the Doppler-shifted modulation band on the slow-time frequency spectrum (Fig. $9(\mathrm{~g}))$. This contrast-to-tissue ratio lost was certainly involved by microbubbles decorrelation due to the necessary decrease in total frame rate as the number if plane-wave compounding angles was increased. Indeed, in this case, signal interferes destructively during the RF summation across transmit angles for moving bubbles but not stationary tissue [43]. Note that plane-wave compounding may also lead to blur motion which could also impact the contrast-to-tissue ratio. Thus, according to the expected flow speed, the number of angles has to be chosen carefully to avoid a decorrelation effect. In presence of flow, the number of angles and the number of microbubble modulation-states have to be chosen to avoid an overlap of the Doppler shifts from the modulation frequency and its multiple.

The use of a lock-in amplifier (selective bandpass filter combined with a demodulation stage) instead of a selective bandpass filter without demodulation stage allowed to increase the relative contrast-to-tissue ratio from 26 to $48 \%$ on average (examples are presented on Fig. 12), and up to 65\%. The lock-in amplifier was particularly interesting for the low amplitudes of the modulation excitation, a limited number of compounding angles and modulation states, along with the slow flow. Moreover, the microbubbles videos obtained after demodulation with the lock-in amplifier presented fewer artifacts as compared to a simple bandpass filter. However, it has to be noted that the processing time with the lock-in amplifier is around 3 times higher than with a bandpass filter (20 s for one angle for the lock-in amplifier instead of $7 \mathrm{~s}$ for the bandpass filter). The minimum number of images for the lock-in 
amplifier (or bandpass filter) was 144 (defined by the Matlab functions used). There were no effect on microbubbles contrast of the number of images in the lock-in amplifier filter process (Fig. 13(a)). In in vivo conditions, with tissue displacement in addition to microbubbles displacements, the limitation of the number of images was expected to lead to less efficient filtering of the lock-in amplifier and so to a decrease of microbubbles contrast. However, for future clinical implementation, the number of images should be optimzed as a function of the number of modulation states, angles and imaging depth, to attain sufficient image repetition frequency. Nonetheless, the increase in the number of images led to an increase of microbubbles contrast (Fig. 13(b)).

In a flow phantom condition without flow, ultrafast acquisitions may not be necessary and the use of conventional acquisitions with a lower frame rate should lead to a similar contrast-to-tissue ratio. However, even for a very low flow speed, conventional acquisitions may lead to microbubble decorrelation and then, to a decrease of the contrast-to-tissue ratio. In in vivo conditions, tissue displacement could pollute the spectrum in the slow time (the flow effect corresponds to the first part of the spectrum in the slow time and is more prominent for higher flow speed (Fig. 7(f)) and lower frame rate (Fig. 9(f))). So, using a conventional acquisition in in vivo conditions could lead to a decrease in contrast-to-tissue ratio.

In addition to simplifying the process, the combination of RMI with ultrafast acquisition allowed to increase the sensitivity of this technique for several reasons. First, this process allowed to study more microbubble modulation-states than classical RMI and the contrast-to-tissue ratio increased slightly with the number of microbubble modulation-states. Moreover, it allowed the use of the multi-band uRMI which increased drastically the contrast-to-tissue ratio (Fig. 8(a)). The lock-in amplifier itself was responsible for a substantial increase in the contrast-to-tissue ratio (Fig. 12). Finally, ultrafast acquisitions by reducing the impact of the displacement on the spectrum in the slow time should prevent a decrease in the contrast-to-tissue ratio.

In absence of flow, uRMI provided the best contrast-to-tissue ratio compared to SVD spatiotemporal filter, amplitude modulation and microbubbles disruption (Fig. 11(a)). Amplitude modulation was expected to provide a lower contrast-to-tissue ratio than RMI from simulation (Fig. 3), as the nonlinear contribution to microbubbles scattering is expected to be very small at 15 MHz. It was confirmed by the experimental results where the contrast-to-tissue ratio was found to be lower with amplitude modulation than with uRMI (Fig. 11). As compared to amplitude modulation, uRMI performed very well at high frequency. Amplitude modulation was applied in several studies which showed enhancement of $14 \mathrm{~dB}$ at $2.5 \mathrm{MHz}$ [14] compared to linear excitation or a contrast-to-tissue ratio of $15 \mathrm{~dB}$ at $8 \mathrm{MHz}$ after state lag correction [44] and $13 \mathrm{~dB}$ at 18-24 MHz [45]. In our study, for consistency, amplitude modulation was applied for the same imaging voltage than uRMI and the contrast-to-tissue ratio was between 8 and $10 \mathrm{~dB}$ at $15 \mathrm{MHz}$ in these conditions. This difference with Needles' study [45], may come from the lower microbubbles concentration $\left(3 \times 10^{4}-16 \times 10^{4}\right.$ microbubbles $/ \mathrm{mL}$ compared to $4 \times 10^{6}$ microbubbles $\left./ \mathrm{mL}\right)$ or a difference in imaging pressure (300 kPa compared to 200 to $500 \mathrm{kPa}$ ). Note that, in contrary to amplitude modulation, the implementation of RMI leverages significant SNR gain from the Doppler ensemble. The contrast-to-tissue ratio that may be obtained for microbubbles 
disruption without flow was found to be close to $6 \mathrm{~dB}$. This contrast, as expected from simulation (Fig. 3), was better than amplitude modulation. However, according to numerical simulation (Fig. 3), microbubbles disruption was expected to give a higher contrast-to-tissue ratio than RMI. The low contrast-to-tissue ratio obtained with microbubbles disruption (presence or not of microbubbles) may be explained by the noise from the phantom in the vessel due to the limited number of angles used (only 3 ). Ultrafast RMI is then more appropriate to detect stopped microbubbles than the other tested techniques in this study to realized ultrasound molecular imaging acquisitions.

With a flow speed from 0.01 to $5 \mathrm{~mL} / \mathrm{min}$ (corresponding to 0.05 to $25 \mathrm{~mm} / \mathrm{s}$ microbubble speed in the center of the canal), contrast-to-tissue ratios obtained with SVD spatiotemporal filter increased with the flow speed. It was in agreement with the observation of Desailly's study [24]. For these flow speed, contrast-to-tissue ratios obtained with uRMI were stable and so uRMI allow a better contrast than SVD filtering for flow speed $\leq 0.05 \mathrm{~mL} / \mathrm{min}(\leq 0.25 \mathrm{~mm} / \mathrm{s})$. Then uRMI should be appropriate to detect the very slowly moving microbubbles in the finest vessels. SVD filtering and uRMI may be complementary techniques to retrieve the most complete as possible microvascular cartographies: microbubbles moving faster than $1 \mathrm{~mm} / \mathrm{s}$ may be detected with SVD filtering and microbubbles moving lower may be detected with uRMI.

The experiment and uRMI, in general, suffer from some drawbacks. Firstly, for its implementation, we had to couple the imaging $15 \mathrm{MHz}$-transducer with a $1 \mathrm{MHz}$ modulation transducer. However, the later transducer is a single-element narrow-band one, which is a simple technology, easy to produce in any laboratory. A more complex problem is to make sure that the zone to be observed is illuminated by both transducers. Secondly, uRMI is sensitive to the decorrelation of the microbubbles, as in any other nonlinear sequence. But, we already demonstrated that spatiotemporal filtering of ultrafast images outperforms other nonlinear sequences in the presence of flow [24]. Consequently, uRMI can be used as a complementary technique specialized in slow or stopped microbubbles.

\section{CONCLUSION}

In this study, we showed that uRMI increases the contrast-to-tissue ratio at $15 \mathrm{MHz}$ in a phantom vessel without flow and for slow flow $(\leq 0.05 \mathrm{~mL} / \mathrm{min}$ ) compared to SVD filtering. Moreover, in cases of with or without flow, uRMI provides a better contrast-to-tissue ratio than amplitude modulation and microbubble disruption. In future work, the advantage of uRMI should be shown in vivo for ultrasound molecular imaging and ultrasound localization microscopy [21-23] applications. For this last application, uRMI may help to detect microbubbles moving slowly in the finest vessels which were not detected by SVD filtering.

\section{REFERENCES}

[1] K.J.W Taylor, P.N. Burns and P.N.T. Wells, Clinical applications of Doppler ultrasound, Raven Press, New York, NY; 1993. 
[2] M. Tanter, J. Bercoff, L. Sandrin and M. Fink M, " Ultrafast compound imaging for 2-D motion vector estimation: Application to transient elastography ", IEEE transactions on ultrasonics, ferroelectrics, and frequency control. Vol. 49(10), pp. 1363-1374, 2002.

[3] G. Montaldo, M. Tanter, J. Bercoff, N. Benech and M. Fink, "Coherent plane-wave compounding for very high frame rate ultrasonography and transient elastography", IEEE transactions on ultrasonics, ferroelectrics, and frequency control. Vol. 56(3), pp. 489-506. 2009.

[4] J. Bercoff, G. Montaldo, T. Loupa, D Savery, F. Mézière, M. Fink and M. Tanter, "Ultrafast compound Doppler imaging: Providing full blood flow characterization", IEEE Transactions on Ultrasonics, Ferroelectrics and Frequency Control. Vol. 58(1), pp. 134-147, 2011.

[5] M. Tanter and M. Fink, "Ultrafast imaging in biomedical ultrasound", IEEE transactions on ultrasonics, ferroelectrics, and frequency control. Vol. 61(1), pp. 102-119. 2014.

[6] E. Macé, G. Montaldo, I. Cohen, M. Baulac, M. Fink and M. Tanter, "Functional ultrasound imaging of the brain", Nature Methods. Vol. 8(8), pp. 662-666, 2011.

[7] T. Deffieux, C. Demene, M. Pernot and M. Tanter, "Functional ultrasound neuroimaging: a review of the preclinical and clinical state of the art", Current opinion in neurobiology. Vol. 50, pp. 128-135, 2018.

[8] R. Gramiak and P.M. Shah, "Echocardiography of the aortic root", Investigative Radiology. Vol 3, pp. 356-366. 1968.

[9] P.N. Burns and S.R. Wilson, "Microbubble contrast for radiological imaging: 1. Principles", Ultrasound quarterly. Vol. 22(1), pp. $5-13,2006$.

[10] N. De Jong, A. Bouakaz and P. Frinking, "Basic acoustic properties of microbubbles", Echocardiography. Vol. 19(3), pp. 229-240, 2002.

[11] C.X. Deng and F.L. Lizzi, "A review of physical phenomena associated with ultrasonic contrast agents and illustrative clinical applications", Ultrasound in medicine \& biology. Vol. 28(3), pp. 277-286, 2002.

[12] Means for increasing sensitivity in non-linear ultrasound imaging systems, by G.A. Brock-Fisher, M.D. Poland and P.G. Rafter. (1996, November 26). Patent US5577505A.

[13] E. G. Caiani, K.A. Collins, C.E. Korcarz, J.E. Bednar, R.M. Lang and others, "Combined assessment of myocardial perfusion and regional left ventricular function by analysis of contrast-enhanced power modulation images", Circulation. Vol. 4, pp. 352-357, 2001.

[14] R.J. Eckersley, C.T. Chin and P.N. Burns, "Optimising phase and amplitude modulation schemes for imaging microbubble contrast agents at low acoustic power", Ultrasound in medicine \& biology. Vol. 31(2), pp. 213-219, 2005.

[15] O. Couture, S. Bannouf, G. Montaldo, J-F. Aubry, M. Fink and M. Tanter. "Ultrafast imaging of ultrasound contrast agents". Ultrasound in medicine \& biology, 35(11), pp.1908-1916, 2009. 
[16] O. Couture, M. Fink and M. Tanter. "Ultrasound contrast plane wave imaging." IEEE transactions on ultrasonics, ferroelectrics, and frequency control, Vol. 59(12): 2676-2683, 2012.

[17] C. Tremblay-Darveau, R. Williams, L. Milot, M. Bruce and P. N. Burns. "Combined perfusion and doppler imaging using plane-wave nonlinear detection and microbubble contrast agents". IEEE transactions on ultrasonics, ferroelectrics, and frequency control, 61(12), pp.1988-2000, 2014.

[18] F. Forsberg, W.T. Shi and B.B. Goldberg, "Subharmonic imaging of contrast agents", Ultrasonics. Vol. 38(1-8), pp. 93-98, 2000.

[19] D.E. Goertz, E. Cherin, A. Needles, R. Karshafian, A.S. Brown, P.N. Burns and F.S. Foster, "High frequency nonlinear B-scan imaging of microbubble contrast agents", IEEE transactions on ultrasonics, ferroelectrics, and frequency control. Vol. 52(1), pp. 65-79, 2005.

[20] R.C. Gessner, C.B. Frederick, F.S. Foster and P.A. Dayton, "Acoustic angiography: a new imaging modality for assessing microvasculature architecture", Journal of Biomedical Imaging. Vol. 2013, ID 936593. 2013

[21] O. Couture, B. Besson, G. Montaldo, M. Fink and M. Tanter, "Microbubble ultrasound super-localization imaging (MUSLI)", In Ultrasonics Symposium, Orlando, FL, USA, 2011, pp. 1285-1287.

[22] C. Errico, J. Pierre, S. Pezet, Y. Desailly, Z. Lenkei, O. Couture and M. Tanter, "Ultrafast ultrasound localization microscopy for deep super-resolution vascular imaging", Nature. Vol. 527(7579), pp. 499-502, 2015.

[23] O. Couture, V. Hingot, B. Heiles, P. Muleki-Seya and M. Tanter, "Ultrasound localization microscopy and super-resolution: A state of the art", IEEE transactions on ultrasonics, ferroelectrics, and frequency control. Vol. 65(8), pp. 1304-1320, 2018.

[24] Y. Desailly, A-M. Tissier, J-M. Correas, F. Wintzenrieth, M. Tanter and O. Couture, "Contrast enhanced ultrasound by real-time spatiotemporal filtering of ultrafast images", Physics in Medicine \& Biology. Vol. 62(1), pp. 31-42. 2017.

[25] D.B. Ellegala, J.E. Carpenter, A.L. Klibanov, M.E. Shaffrey, J.R. Lindner and others, "Imaging tumor angiogenesis with contrast ultrasound and microbubbles targeted to $\alpha v \beta 3 "$, Circulation. Vol. 108, pp. 336-341. 2003.

[26] G.E.R. Weller, M.K.K. Wong, R.A. Modzelewski, E. L, A.L. Klibanov, W.R. Wagner and F.S. Villanueva, "Ultrasonic imaging of tumor angiogenesis using contrast microbubbles targeted via the tumor-binding peptide arginine-arginine-leucine", Cancer research. Vol. 65(2), pp. 533-539, 2005.

[27] J.K. Willman, R. Paulmurugan, K. Chen, O. Gheysens, M. Rodriguez-Porcel, A.M. Lutz, I.Y. Chen, X. Chen and S.S. Gambhir, "US imaging of tumor angiogenesis with microbubbles targeted to vascular endothelial growth factor receptor type 2 in mice", Radiology. Vol. 246(2), pp. 508-518, 2008.

[28] P. Marmottant, S. van derMeer, M. Emmer, M. Versluis, N. de Jong, S. Hilgenfeldt and D. Lohse, "A model for large amplitude oscillations of coated bubbles accounting for buckling and rupture", Journal of Acoustical Society of America. Vol. 118(6), pp. 3499-3505, 2005. 
[29] C.X. Deng, F.L. Lizzi, A. Kalisz, A. Rosado, R.H. Silverman and D.J. Coleman, "Study of ultrasonic contrast agents using a dual-frequency band technique", Ultrasound in medicine \& biology. Vol. 26(5), pp. 819-831, 2000.

[30] A. Bouakaz and N. de Jong, "New contrast imaging method using double frequency exposure", In Ultrasonics Symposium, Montreal, QC, Canada, 2004, pp. 339-342.

[31] M. Emmer, H.J. Vos, M. Versluis and N. De Jong, "Radial modulation of single microbubbles", IEEE transactions on ultrasonics, ferroelectrics, and frequency control. Vol. 56(11), pp 2370-2379, 2009.

[32] E. Chérin, J. Brown, S-E. Måsøy, H. Shariff, R. Karshafian, R. Williams, P.N. Burns and F.S. Foster, "Radial modulation imaging of microbubble contrast agents at high frequency", Ultrasound in medicine \& biology. Vol. 34(6), pp. 949-962, 2008.

[33] H.H. Shariff, P.D. Bevan, R. Karshafian, R. Williams and P.N. Burns, "Radial Modulation Imaging: Raising the Frequency for Contrast Imaging", In Ultrasonics Symposium, Vancouver, BC, Canada, 2006, pp. 104-107.

[34] B.A. Angelsen and R. Hansen, "SURF Imaging-A new method for ultrasound contrast agent imaging", In Ultrasonics Symposium, New-York, NY, USA, 2007, pp. 531-541.

[35] R. Hansen and B.A. Angelsen, "SURF imaging for contrast agent detection", IEEE transactions on ultrasonics, ferroelectrics, and frequency control. Vol. 56(2), pp. 280-290, 2009.

[36] R. Hansen, S-E. Måsøy, T.A. Tangen and B.A. Angelsen, "Nonlinear propagation delay and pulse distortion resulting from dual frequency band transmit pulse complexes", The Journal of the Acoustical Society of America. Vol. 129(2), pp. 1117-1127, 2011.

[37] F.T.H. Yu, F.S. Villanueva and X. Chen, "Radial modulation contrast imaging using a 20-MHz single-element intravascular ultrasound catheter", IEEE transactions on ultrasonics, ferroelectrics, and frequency control. Vol. 61(5), pp. 779-791, 2014.

[38] S-E. Masoy, O. Standal, P. Nasholm, T.F. Johansen, B.A. Angelsen and R. Hansen, "SURF imaging: In vivo demonstration of an ultrasound contrast agent detection technique", IEEE transactions on ultrasonics, ferroelectrics, and frequency control. Vol. 55(5), pp. 1112-1121. 2008 .

[39] J. Bercoff, G. Montaldo, T. Loupas, D. Savery, F. Mézière, M. Fink and M. Tanter, "Ultrafast compound Doppler imaging: Providing full blood flow characterization", IEEE Transactions on Ultrasonics, Ferroelectrics and Frequency Control. Vol. 58(1), pp. 134-147, 2011.

[40] L. Hoff, Acoustic characterization of contrast agents for medical ultrasound imaging, Springer Science \& Business Media; 2001.

[41] M. Schneider, "Characteristics of SonoVuetrade mark", Echocardiography. Vol. 16(7), pp. 743-746, 1999.

[42] C. Barriere and D. Royer, "Diffraction effects in the parametric interaction of acoustic waves: application to measurements of the nonlinearity parameter B/A in liquids". IEEE transactions on ultrasonics, ferroelectrics, and frequency control, 48(6), pp.1706-1715, 2001. 
[43] B. Denarie, T. A. Tangen, I. K. Ekroll, N. Rolim, H. Torp, T. Bjastad and L. Lovstakken, "Coherent plane wave compounding for very high frame rate ultrasonography of rapidly moving targets", IEEE transactions on medical imaging. Vol. 32(7), pp. 1265-1276, 2013.

[44] C. Tremblay-Darveau, P.S. Sheeran, C.K. Vu, R. Williams, Z. Zhang, M. Bruce and P.N. Burns, "The role of microbubble echo phase lag in multi-pulse contrast-enhanced ultrasound imaging", IEEE Transactions on Ultrasonics, Ferroelectrics, and Frequency Control. Vol. 65(8), pp 1389-1401, 2018.

[45] A. Needles, M. Arditi, N.G. Rognin, J. Mehi, T. Coulthard, C. Bilan-Tracey, E. Gaud, P. Frinking, D. Hirson and F.S. Foster, "Nonlinear contrast imaging with an array-based micro-ultrasound system", Ultrasound in medicine \& biology. Vol. 36(12), pp. 2097-2106, 2010. 
TABLE I

THE CONTRAST-TO-TISSUE RATIO BETWEEN MICROBUBBLES IN THE VESSEL AND THE PHANTOM FOR A 3 ANGLES HIGH-FREQUENCY EXCITATION TO EVALUATE THE MODULATION/DEMODULATION PROCESS: WITH AND WITHOUT MICROBUBBLES AND WITH AND WITHOUT A LOW-FREQUENCY EXCITATION (4 MICROBUBBLE MODULATION-STATES, $15 \mathrm{KPA})$.

\begin{tabular}{lcc}
\hline \hline Microbubbles/Phantom contrast & uRMI (dB) & Bmode (dB) \\
\hline No microbubble, no low frequency & -0.4 & -7.0 \\
No microbubble, low frequency & 2.4 & -7.0 \\
Microbubble, no low frequency & -0.5 & -3.8 \\
Microbubble, low frequency & 12.7 & -3.5 \\
\hline
\end{tabular}




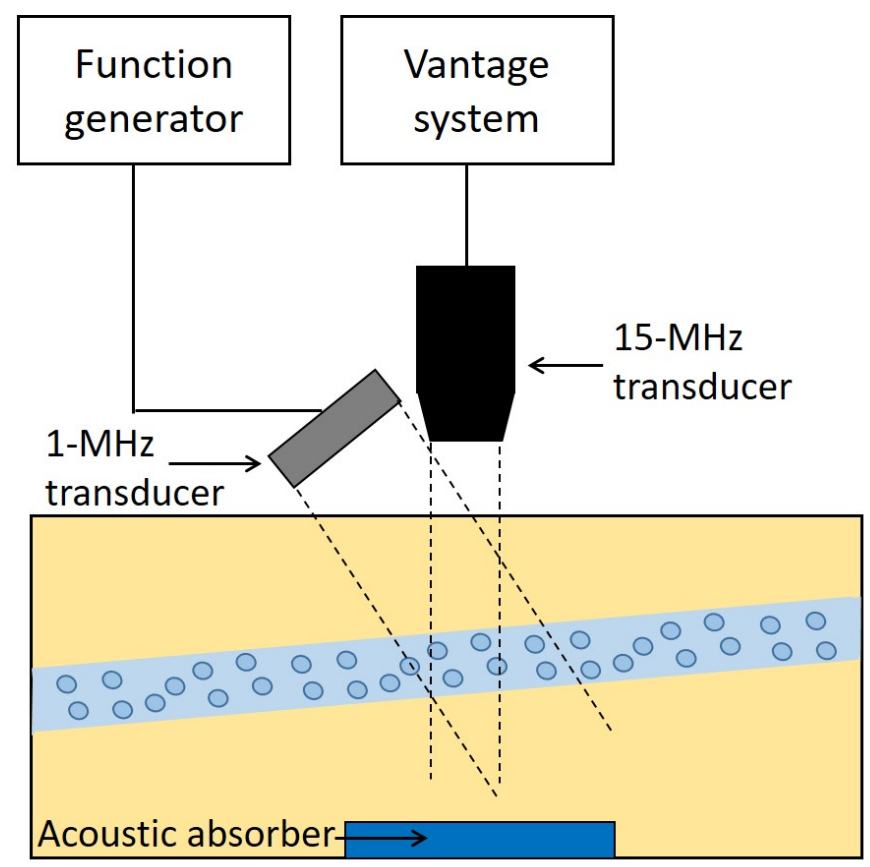

Fig. 1. Experimental setup: the 1-MHz transducer, piloted by the function generator, and the $15-\mathrm{MHz}$ transducer, piloted by the vantage system, were placed above the flow phantom, in a way that their pressure fields overlapped at the vessel phantom location. 

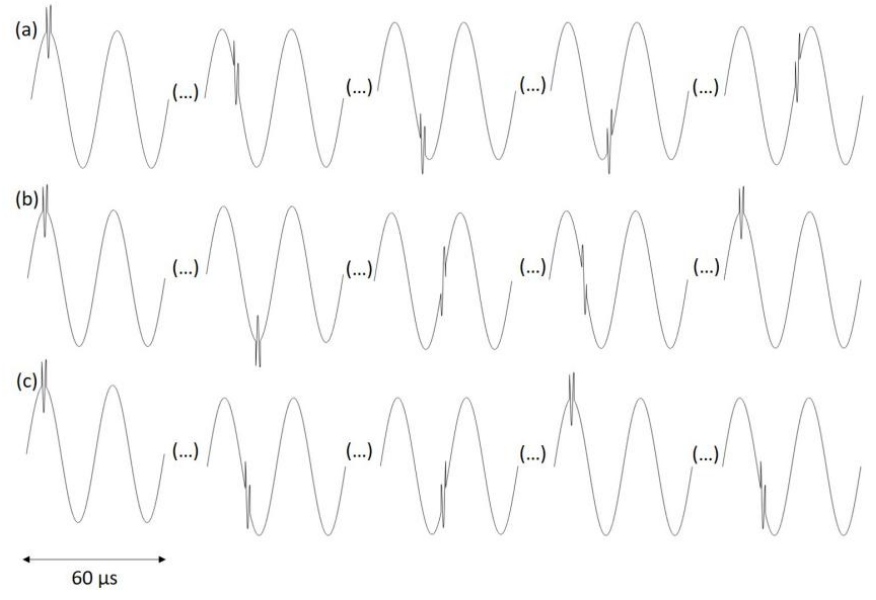

Fig. 2. Illustration of RMI pulses with 5 (a), 4 (b) or 3 (c) microbubble modulation-states per period. The manipulation pulse is a continuous sinusoidal signal with the low-frequency transducer and the imaging pulse is a 1 cycle $15 \mathrm{MHz}$ pulse every $60 \mu \mathrm{s}$. 

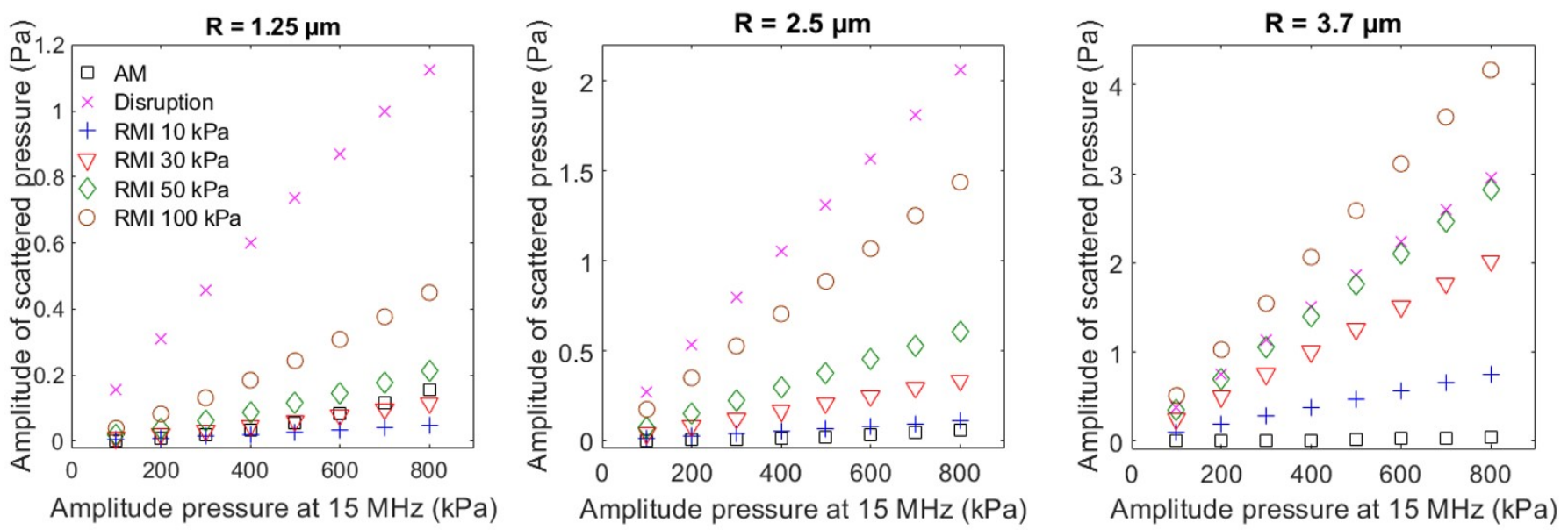

Fig. 3. Scattered pressure from a microbubble of $1.25 \mu \mathrm{m}, 2.5 \mu \mathrm{m}$ and $3.7 \mu \mathrm{m}$ radius in amplitude modulation (MA) imaging, microbubble disruption and RMI as a function of the amplitude pressure at $15 \mathrm{MHz}$. The RMI results are presented for 4 amplitudes pressure of the $1 \mathrm{MHz}$ excitation. 

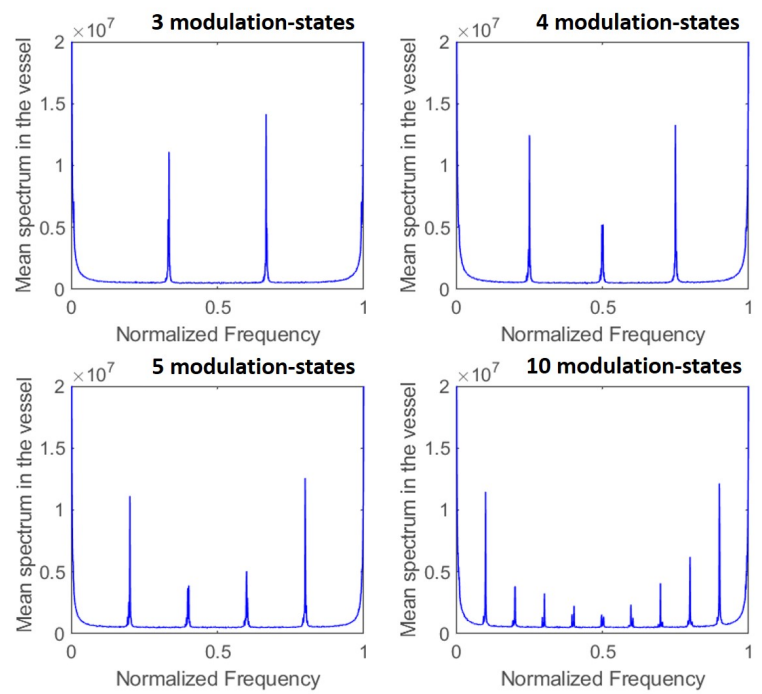

Fig. 4. Example of normalized frequency spectrums in slow time (divided by the sampling frequency fs) for 3, 4, 5 and 10 microbubble modulation-states. 

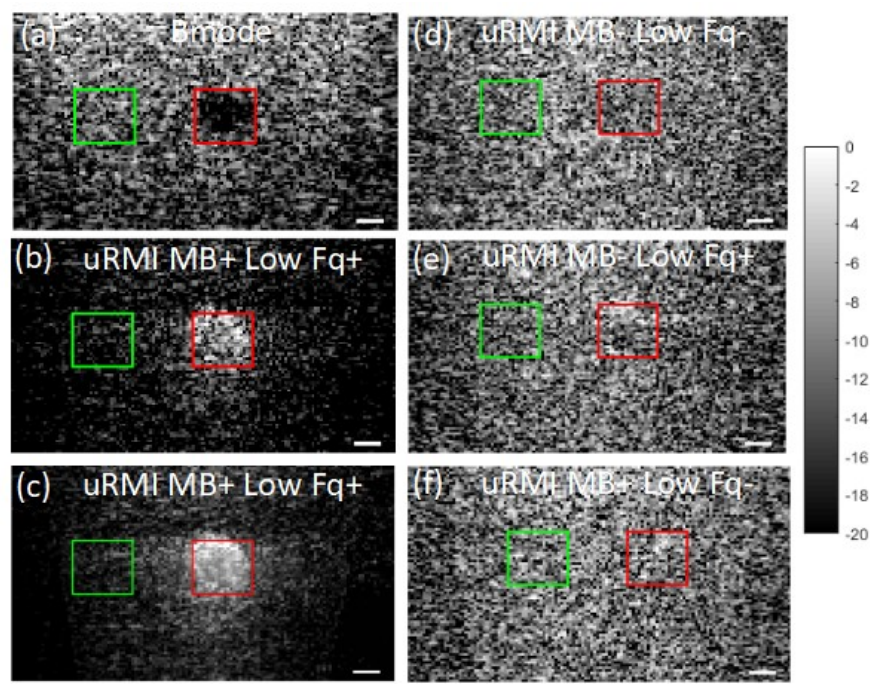

Fig. 5. Examples of images for a 3 angles, 4 microbubble modulation-states and $15 \mathrm{kPa}$ acquisition without flow: (a) one Bmode image with microbubbles, (b) one uRMI image with microbubbles and with the low-frequency excitation, (c) sum of 1000 uRMI images with microbubbles and with the low-frequency excitation, (d) one uRMI image without microbubbles without low-frequency excitation, (e) one uRMI image without microbubbles with low-frequency excitation, and (f) one uRMI image with microbubbles without low-frequency excitation. The red and green rectangles correspond to the ROI of the vessel phantom and the agar, respectively. The scale bar represents $1 \mathrm{~mm}$ and the colorbar is in dB. 

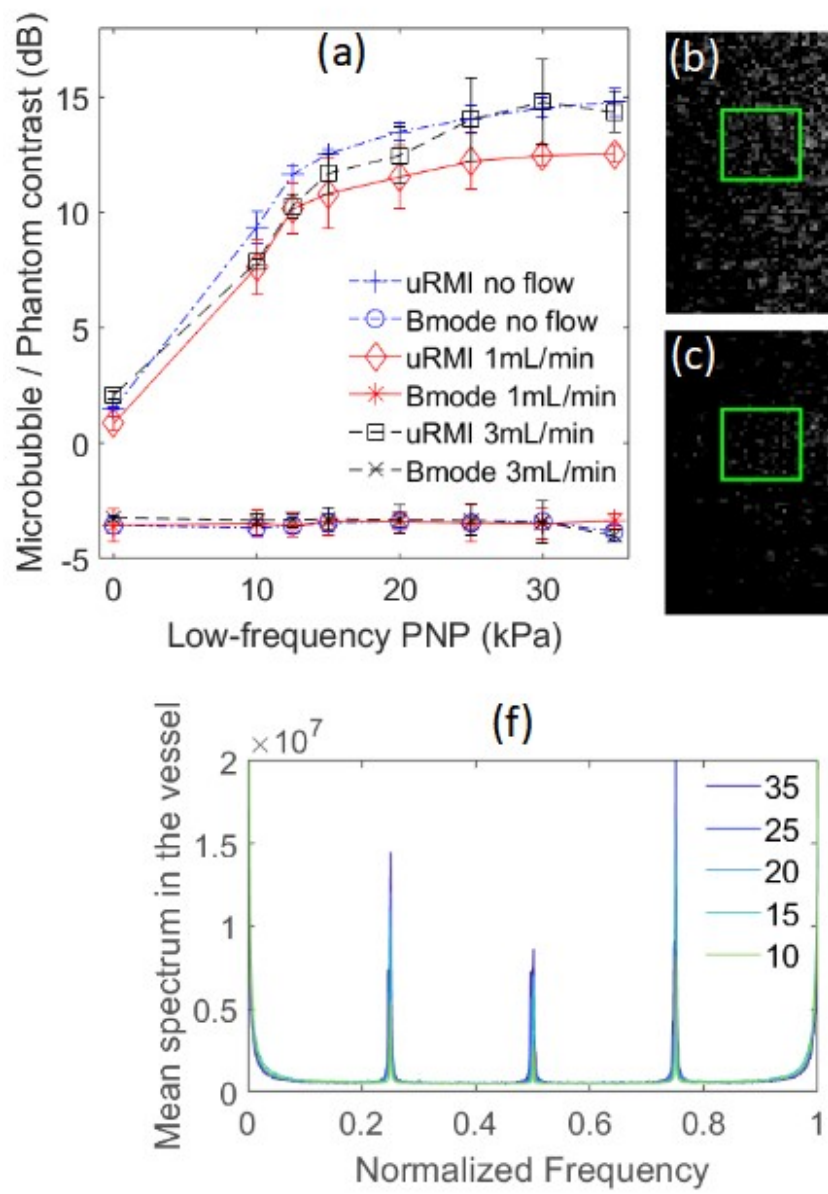
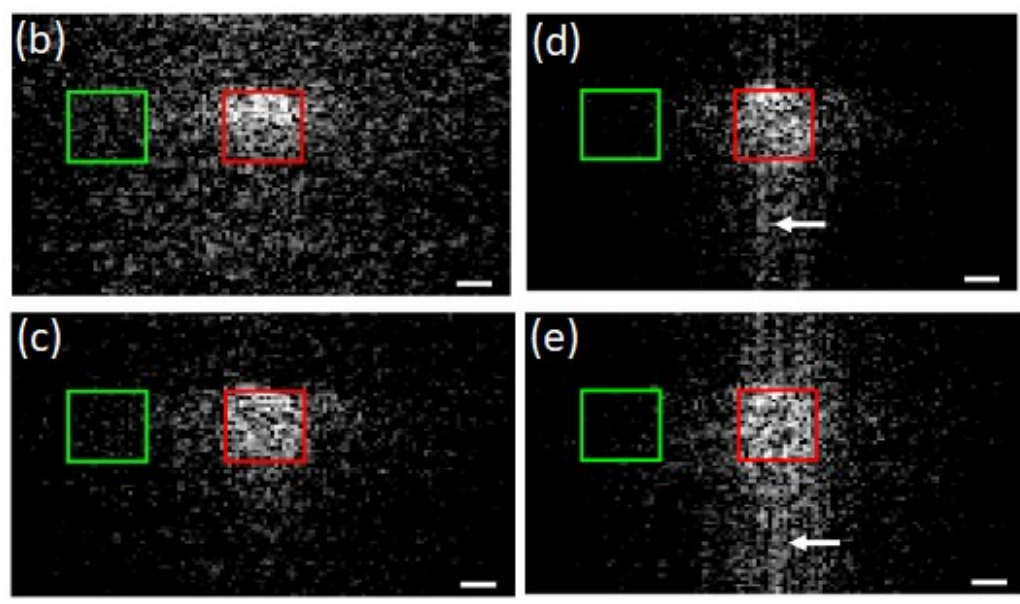

(e)

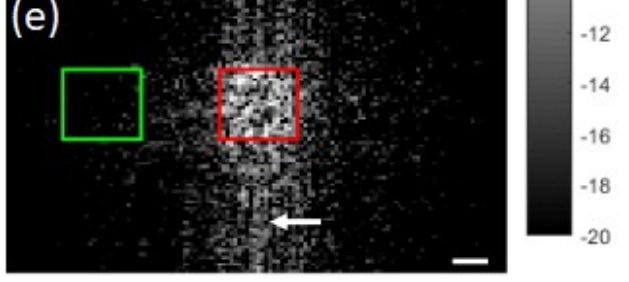

(g)

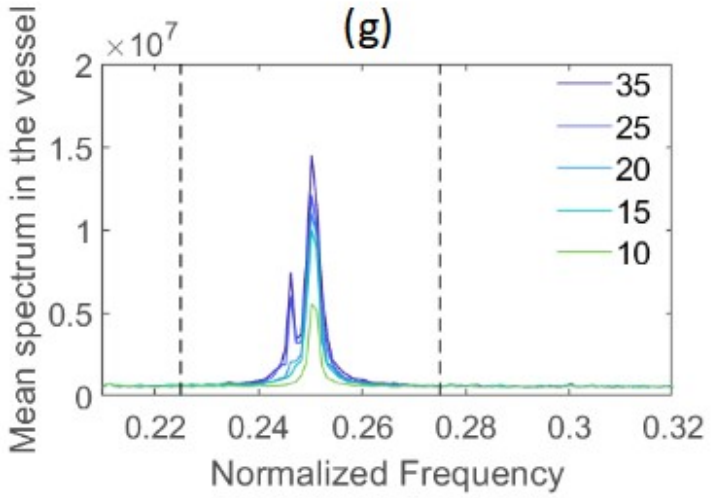

Fig. 6. The contrast-to-tissue ratio between microbubbles in the vessel and the phantom as a function of the amplitude of the $1 \mathrm{MHz}$ modulation pulse for 3 angles, 4 modulation-states acquisition without flow and for a 1 and $3 \mathrm{~mL} / \mathrm{min}$ flow (a) and examples of images for 15 (b), 20 (c), 30 (d) and 35 (e) kPa without flow. The red and green rectangles correspond to the ROI of the vessel phantom and the agar, respectively. White arrows show examples of artifacts observed. The scale bar represents $1 \mathrm{~mm}$ and the colorbar is in $\mathrm{dB}$. Corresponding normalized frequency spectrums in slow time (divided by the sampling frequency fs) of images data-set at the vessel phantom location (f) zoomed around the modulation frequency (g) showing a split peak. The dotted lines show the limits of the bandpass filter of the demodulation process. 

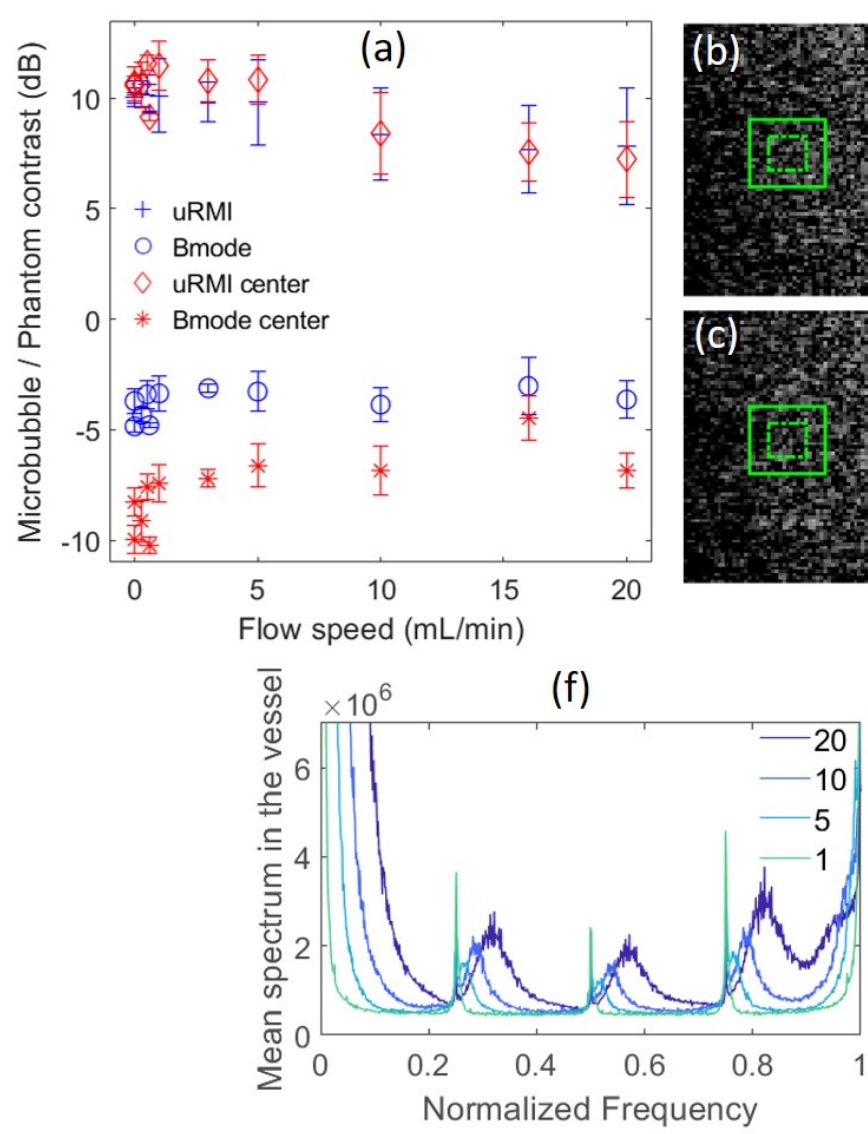
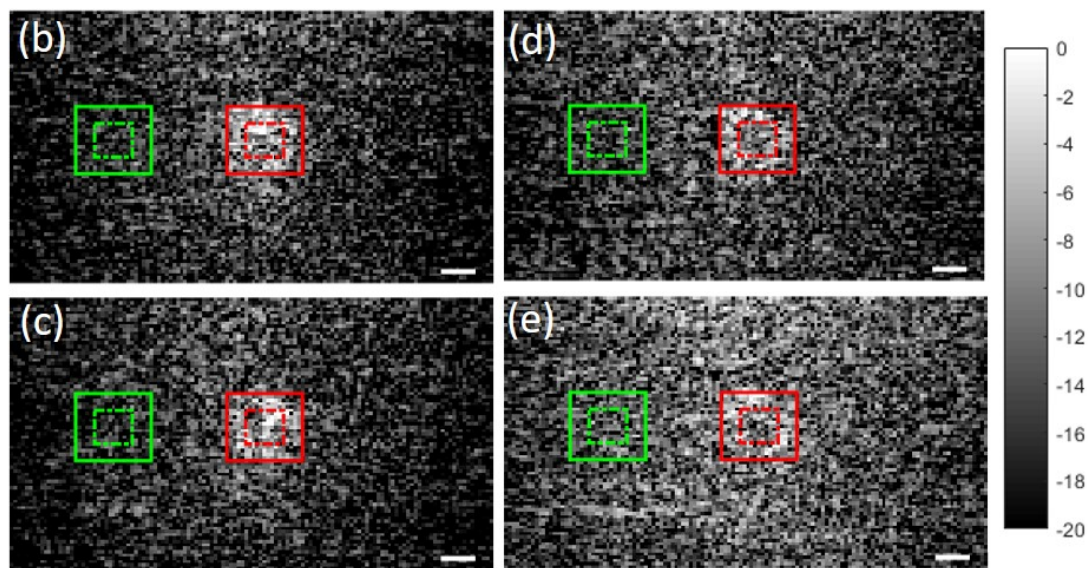

(g)

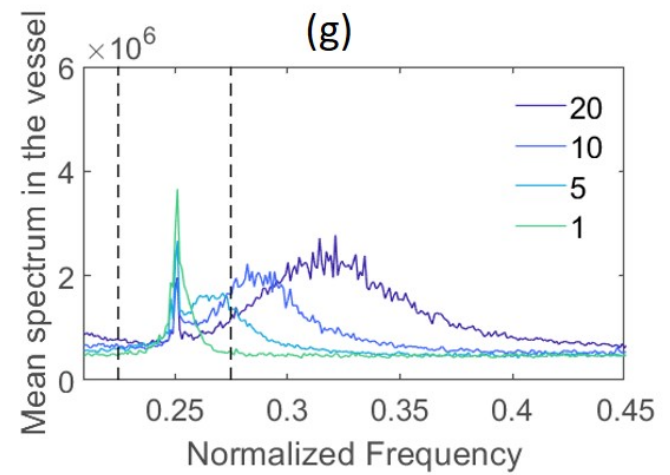

Fig. 7. The contrast-to-tissue ratio between microbubbles in the vessel and the phantom as a function of the flow speed for 3 angles, 4 modulation-states, $15 \mathrm{kPa}$ low-frequency acquisition (a) and examples of images for 1 (b), 5 (c), 10 (d) and 20 (e) $\mathrm{mL} / \mathrm{min}$. The plain red and green rectangles correspond to the ROI of the vessel phantom and the agar, respectively. The dotted red and green rectangles correspond to the ROI in the center of the vessel phantom and the agar, respectively. The scale bar represents $1 \mathrm{~mm}$ and the colorbar is in $\mathrm{dB}$. Corresponding normalized frequency spectrums in slow time (divided by the sampling frequency fs) of images data-set at the vessel phantom location (f) zoomed around the modulation frequency (g). The dotted lines show the limits of the bandpass filter of the demodulation process. 


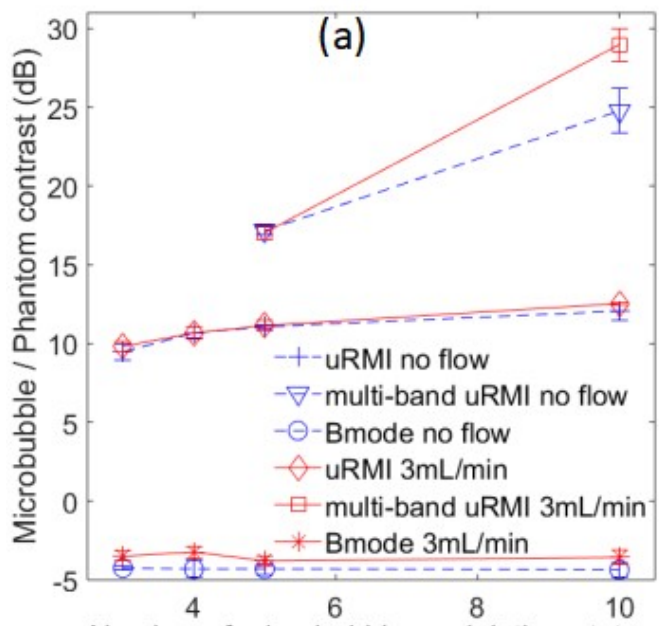

Number of microbubble modulation-states
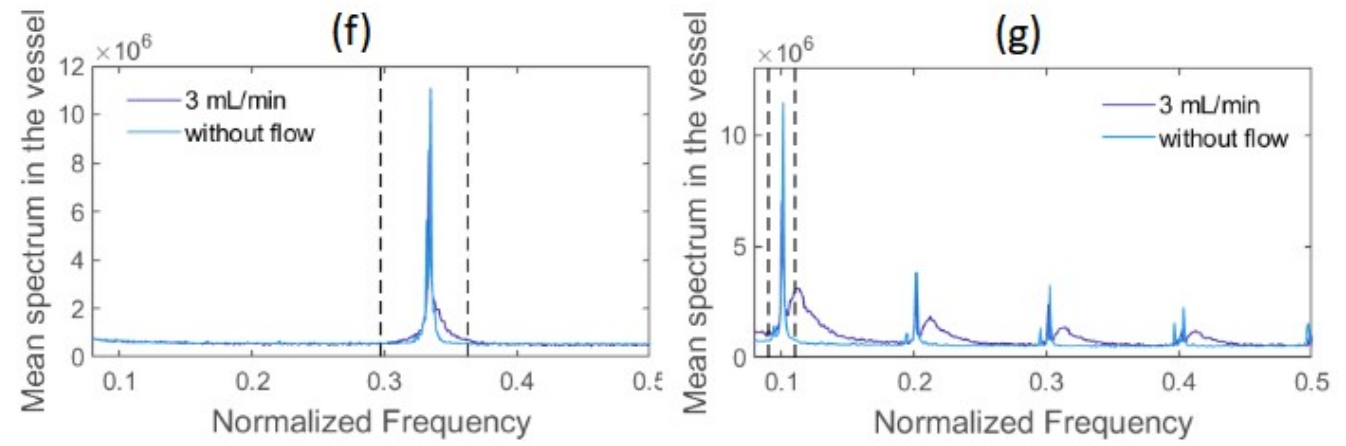

Fig. 8. (a) The contrast-to-tissue ratio between microbubbles in the vessel and the phantom as a function of microbubble modulation-states number for 3 angles, $15 \mathrm{kPa}$ low-frequency acquisition without flow and for a $3 \mathrm{~mL} / \mathrm{min}$ flow speed. Examples of uRMI images for a $3 \mathrm{~mL} / \mathrm{min}$ flow speed for $5 \mathrm{modulation}$-states (b) and 10 modulation-states (c) and examples of multi-band uRMI images for 5 modulation-states (d) and 10 modulation-states (e). The red and green rectangles correspond to the ROI of the vessel phantom and the agar, respectively. The scale bar represents $1 \mathrm{~mm}$ and the colorbar is in $\mathrm{dB}$. Corresponding normalized frequency spectrums in slow time (divided by the sampling frequency fs) of images data-set at the vessel phantom location for 3 (f) and 10 ( $\mathrm{g}$ ) microbubble modulation-states. The dotted lines show the limits of the bandpass filter of the demodulation process. 

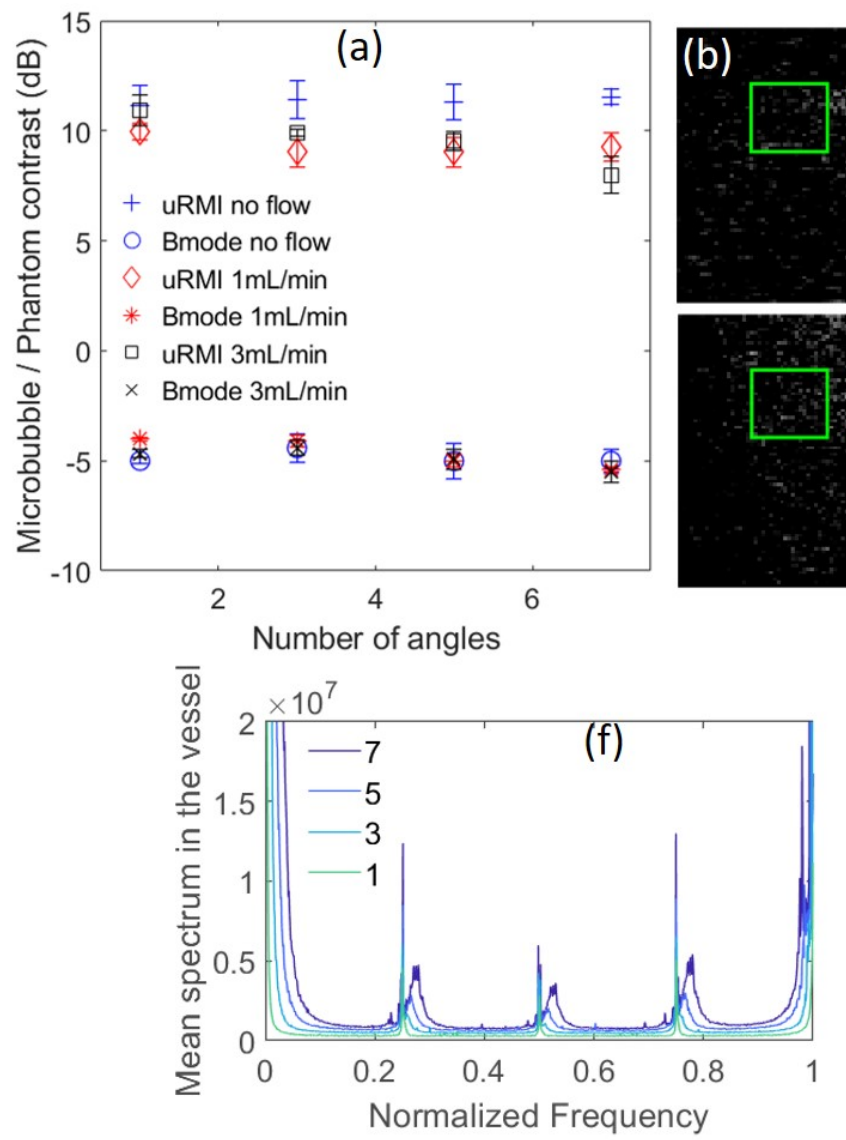
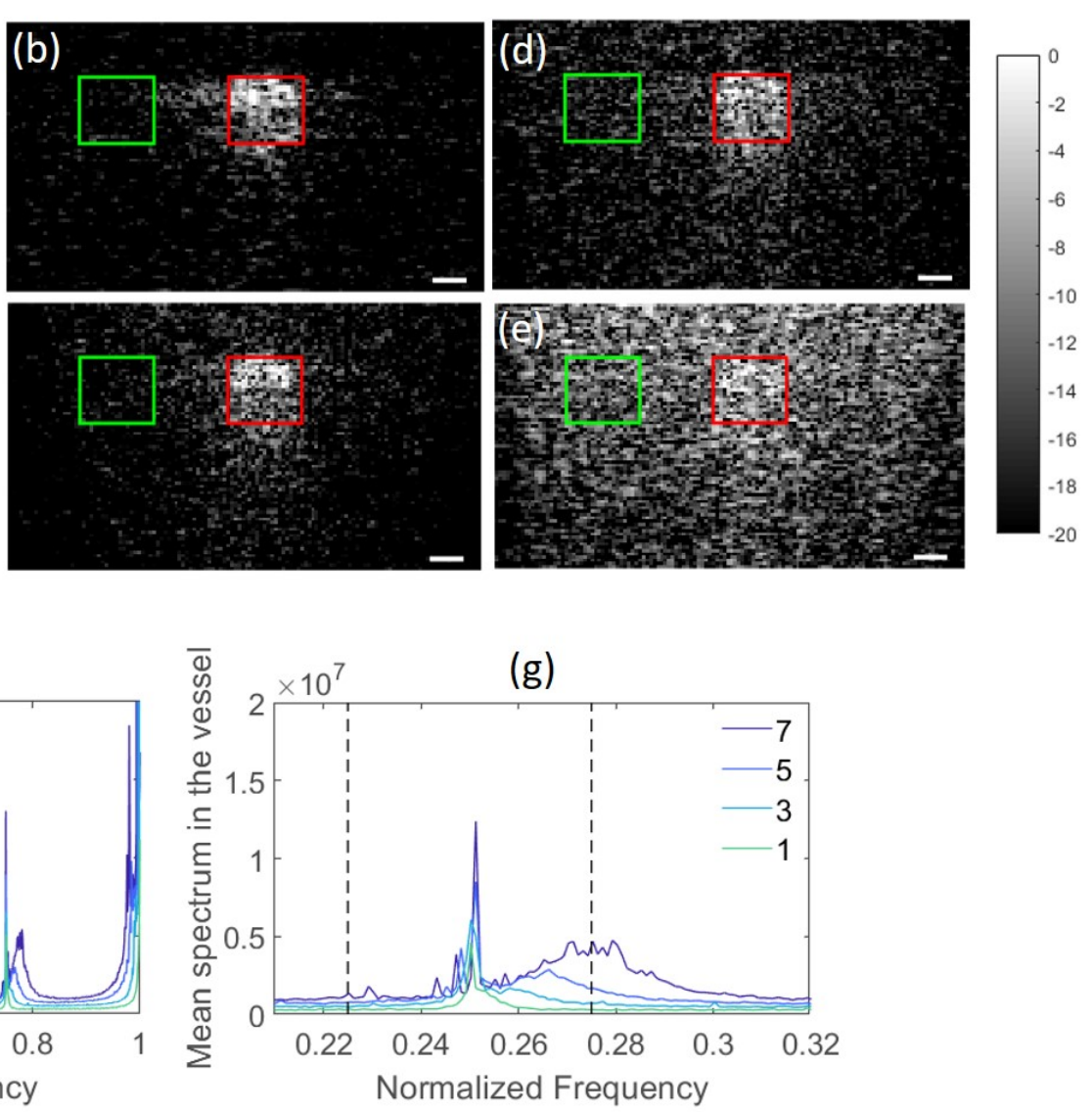

Fig. 9. The contrast-to-tissue ratio between microbubbles in the vessel and the phantom as a function of the number of angles for a 4 modulation-states, $15 \mathrm{kPa}$ low-frequency acquisition without flow and for a $3 \mathrm{~mL} / \mathrm{min}$ flow (a) and examples of images for 1 (b), 3 (c), 5 (d) and 7 (e) angles with a $3 \mathrm{~mL} / \mathrm{min}$ flow speed. The red and green rectangles correspond to the ROI of the vessel phantom and the agar, respectively. The scale bar represents $1 \mathrm{~mm}$ and the colorbar is in $\mathrm{dB}$. Corresponding normalized frequency spectrums in slow time (divided by the sampling frequency fs) of images data-set at the vessel phantom location (f) zoomed around the modulation frequency $(\mathrm{g})$. The dotted lines show the limits of the bandpass filter of the demodulation process. 

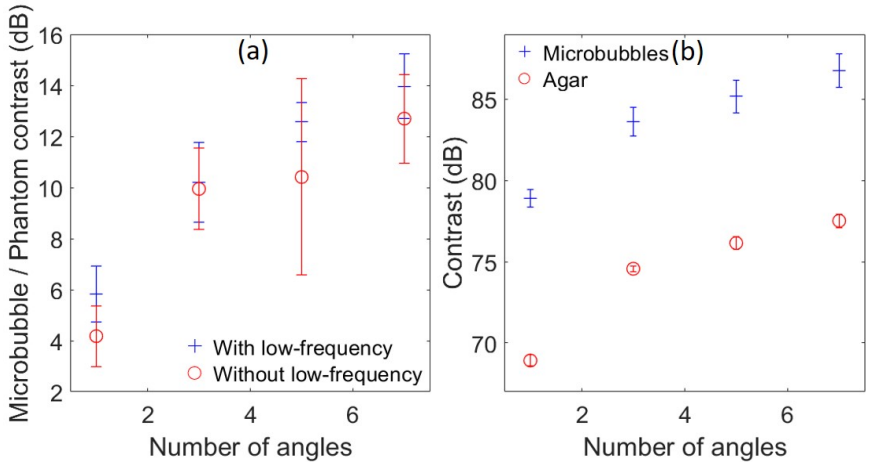

Fig. 10. The contrast-to-tissue ratio between microbubbles in the vessel and the phantom for images-set with and without low-frequency excitation minus its median value over time as a function of the number of angles for 4 microbubble modulation-states, $15 \mathrm{kPa}$ low-frequency acquisition without flow (a). Contrast amplitude of microbubbles in the vessel and amplitude in the phantom without flow as a function of the number of angles for 4 microbubble modulation-states, $100 \mathrm{kPa}$ low-frequency acquisition from uRMI (b). 


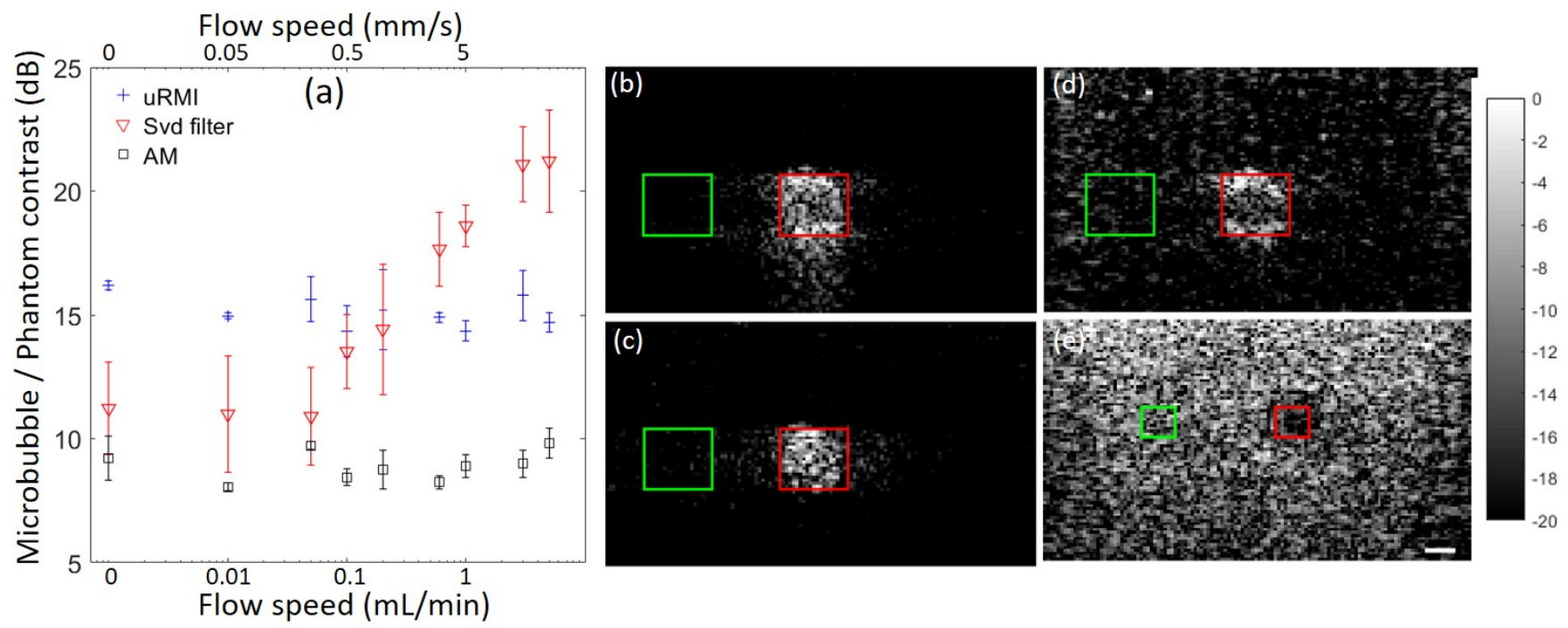

Fig. 11. Contrast-to-tissue ratio between microbubbles in the vessel and the phantom as a function of the flow speed for a 3 angles, 4 modulation-states, 23 $\mathrm{kPa}$ low-frequency acquisition and for amplitude modulation, SVD filtering (a) and examples of images for uRMI (b), SVD filter (c), amplitude modulation for a flow speed of $0.01 \mathrm{~mL} / \mathrm{min}(\mathrm{d})$, and subtraction of an image with microbubbles to an image without microbubbles (e). The red and green rectangles correspond to the ROI of the vessel phantom and the agar, respectively. The scale bar represents $1 \mathrm{~mm}$ and the colorbar is in $\mathrm{dB}$. 

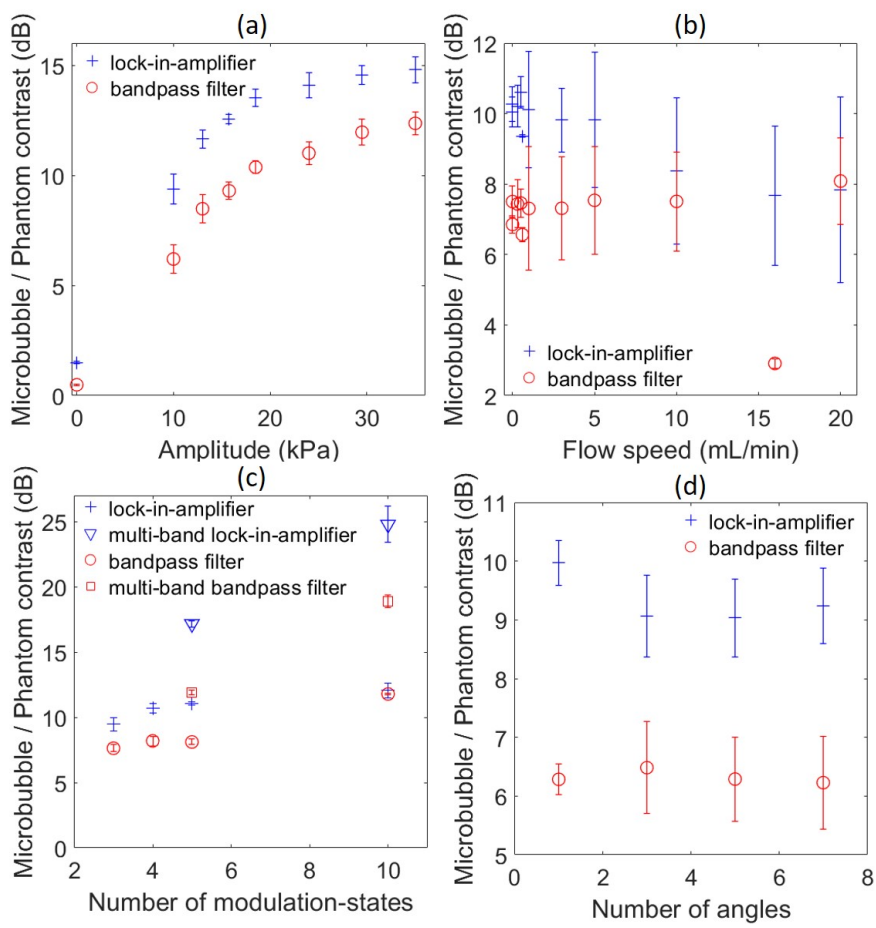

Fig. 12. Contrast-to-tissue ratio between microbubbles in the vessel and the phantom obtained with uRMI and the lock-in-amplifier and with a bandpass filter, as a function of the amplitude of the $1 \mathrm{MHz}$ modulation pulse (a) (3 angles, 4 modulation-states acquisition without flow), of the flow speed (b) (3 angles, 4 modulation-states, $15 \mathrm{kPa}$ low-frequency acquisition), of the number of microbubble modulation-states (c) ( 3 angles, $15 \mathrm{kPa}$ low-frequency acquisition without flow) and of the number of compounding angles (d) (4 modulation-states, $15 \mathrm{kPa}$ low-frequency acquisition, $3 \mathrm{~mL} / \mathrm{min}$ flow speed). 

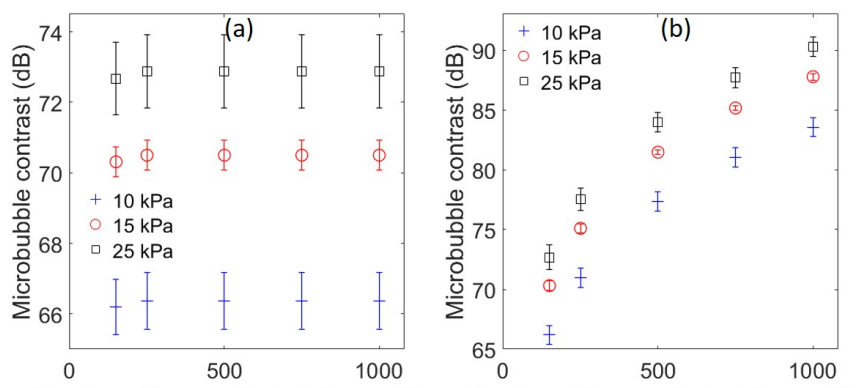

Number of images for lock-in-amplifier Number of images for lock-in-amplifier

Fig. 13. Microbubble contrast as a function of the number of images in the filtering process of the lock-in-amplifier ( 3 imaging angles, 4 microbubble modulation-states, without flow): (a) effect of the number of images on the filtering by evaluating microbubble contrast on the sum of the first 150 images only and (b) effect of the number of images on microbubble contrast by evaluating microbubble contrast on the sum of all images. 